\title{
Solitary excitations in discrete two-dimensional nonlinear Schrodinger models with dispersive dipole-dipole interactions
}

Christiansen, Peter Leth; Gaididei, Yuri Borisovich; Johansson, M.; Rasmussen, Kim; Mezentsev, Vladimir; Juul Rasmussen, Jens

Published in:

Physical Review B Condensed Matter

Link to article, DOI:

10.1103/PhysRevB.57.11303

Publication date:

1998

Document Version

Publisher's PDF, also known as Version of record

Link back to DTU Orbit

Citation (APA):

Christiansen, P. L., Gaididei, Y. B., Johansson, M., Rasmussen, K., Mezentsev, V., \& Juul Rasmussen, J. (1998). Solitary excitations in discrete two-dimensional nonlinear Schrodinger models with dispersive dipoledipole interactions. Physical Review B Condensed Matter, 57(18), 11303-11318.

https://doi.org/10.1103/PhysRevB.57.11303

\section{General rights}

Copyright and moral rights for the publications made accessible in the public portal are retained by the authors and/or other copyright owners and it is a condition of accessing publications that users recognise and abide by the legal requirements associated with these rights.

- Users may download and print one copy of any publication from the public portal for the purpose of private study or research.

- You may not further distribute the material or use it for any profit-making activity or commercial gain

- You may freely distribute the URL identifying the publication in the public portal 


\title{
Solitary excitations in discrete two-dimensional nonlinear Schrödinger models with dispersive dipole-dipole interactions
}

\author{
P. L. Christiansen, Yu. B. Gaididei,* M. Johansson, and K. Ø. Rasmussen \\ Department of Mathematical Modelling, The Technical University of Denmark, DK-2800 Lyngby, Denmark \\ V. K. Mezentsev ${ }^{\dagger}$ and J. Juul Rasmussen \\ Department of Optics and Fluid Dynamics, Risb National Laboratory, DK-4000 Roskilde, Denmark
}

(Received 1 August 1997)

\begin{abstract}
The dynamics of discrete two-dimensional nonlinear Schrödinger models with long-range dispersive interactions is investigated. In particular, we focus on the cases where the dispersion arises from a dipole-dipole interaction, assuming the dipole moments at each lattice site to be aligned either in the lattice plane (anisotropic case) or perpendicular to the lattice plane (isotropic case). We investigate the nature of the linear dispersion relation for these two cases, and derive a criterion for the modulational instability of a plane wave with respect to long-wavelength perturbations. Furthermore, we study the on-site localized stationary states of the system numerically and analytically using a variational approach. In general, the narrow, intrinsically localized states are found to be stable, while broad, "continuumlike" excitations are unstable and may either collapse into intrinsically localized modes or disperse when a small perturbation is applied. [S0163-1829(98)02318-2]
\end{abstract}

\section{INTRODUCTION}

Determination of the dynamical properties of physical systems with competition between discreteness, nonlinearity, and dispersion has attracted a growing interest because of the wide applicability of such models in various physical problems. Examples are coupled optical fibers, ${ }^{1-3}$ arrays of coupled Josephson junctions, ${ }^{4}$ nonlinear charge and excitation transport in biological systems, ${ }^{5}$ and elastic energy transfer in anharmonic crystals. ${ }^{6}$ It has been shown that the balance between nonlinearity and dispersion in a weak nonlinearity (large dispersion) limit provides the existence of low-energy solitonlike excitations. These are very robust objects that propagate essentially without energy loss, and their collisions are almost elastic.

As a result of the interplay between discreteness, dispersion, and nonlinear interactions, new types of nonlinear excitations may appear. These are the intrinsically localized oscillatory states, which are also termed discrete breathers. The properties of these modes have been intensively studied during the past years. ${ }^{1-16}$ For example, in monoatomic lattices with a nearest-neighbor harmonic interaction and a positive quartic anharmonic interaction, localized states with nonlinear frequencies lying above the phonon band were found. ${ }^{10-12}$ In the case of the one-dimensional nonlinear Schrödinger (NLS) lattice ${ }^{17}$ with focusing nonlinearity, there exists below the linear excitation band a localized mode, which in the small amplitude limit reduces to the one-soliton solution of the continuum NLS equation.

A discrete NLS equation with "tunable" diagonal and off-diagonal nonlinearities that includes the integrable Ablowitz-Ladik system ${ }^{7}$ as a limit was introduced in Refs. 18 and 19. It was shown that the reflection and translational symmetries of the integrable equation are broken by the diagonal nonlinearity, and the properties of the Peierls-Nabarro potential as a function of the tuning parameter were studied. Recently, the dynamics in discrete two-dimensional nonlin- ear Schrödinger models with tunable nonlinearities was investigated in Ref. 20. The generalized two-dimensional discrete solitons were found, and their role in the final stage of the quasicollapse dynamics was demonstrated.

Until recently, the main attention was paid to systems with short-ranged dispersive interactions, and a nearestneighbor approximation was used. However, during the last decade a series of theoretical and numerical studies of the effect of long-range interactions on properties of nonlinear excitations was carried out. In Ref. 21 an implicit form for solitons was obtained in a sine-Gordon system with longrange interaction of the Kac-Baker type, ${ }^{22,23}$ and the dependence of the soliton width and energy on the radius of the long-range interaction was analyzed. In Ref. 24 the nonlinear term in the sine-Gordon equation was assumed to have a nonlocal character, and novel soliton states, of topological charge zero, were found to exist at a large enough radius of interaction. In Ref. 25 the effects of a long-range harmonic interaction in a chain with short-range anharmonicity was considered. It was demonstrated that the existence of two velocity-dependent competing length scales leads to two types of solitons with characteristically different width and shapes for two velocity regions separated by a gap. Effects of long-range interactions of the Kac-Baker type were also studied in static and dynamic nonlinear Klein-Gordon ${ }^{26-28}$ and nonlinear Schrödinger ${ }^{29}$ continuum models. In Ref. 30 a one-dimensional discrete NLS model with a power dependence $r^{-s}$ on the distance $r$ of the dispersive interactions was proposed. It was shown that for $s$ sufficiently large, all features of the model are qualitatively the same as in the discrete NLS model with only nearest-neighbor interactions. For $s$ less than a critical value, $s_{\mathrm{cr}} \simeq 3.03$, there is an interval of bistability where for each value of the excitation number two stable stationary states exist: one continuumlike (solitonlike) state and one intrinsically localized (discrete) state.

In the main part of the studies in the literature, the effects of nonlocal interactions were investigated only for one- 
dimensional systems. However, these effects should be of particular importance in systems of higher dimensions, since in $D$-dimensional systems the effective number of atoms that participate in the interaction increases as $R^{D}$ ( $R$ is the radius of the interaction). Moreover, there exist physical situations where the long-range properties of the interactions and the multidimensional character of the systems should be taken into account on the same footing. For example, the excitation transfer in quasi-two-dimensional molecular crystals ${ }^{31}$ and in Langmuir-Blodgett-Scheibe aggregates ${ }^{32}$ is due to transition dipole-dipole interaction with a $r^{-3}$ dependence on the distance $r$. Furthermore, the DNA molecule contains charged groups, with long-range Coulomb interaction $\left(r^{-1}\right)$ between them. Thus, the corresponding vibrational excitation has a dispersive interaction that is also of the dipole-dipole type. As a final example we mention the long-range magnetic order in literally two-dimensional Langmuir-Blodgett films of manganese stearate $\left[\mathrm{Mn}\left(\mathrm{C}_{18} \mathrm{H}_{35} \mathrm{O}_{2}\right)_{2}\right]$, which is stabilized by the magnetic dipole-dipole interaction. ${ }^{33}$

The goal of this paper is to investigate the dynamics in discrete two-dimensional nonlinear Schrödinger models with dipole-dipole dispersive interactions. We also develop a quasicontinuum approach to the problem. The paper is structured in the following way. In Sec. II we define the equations of motions for the system under study and focus our attention on the two particular cases where the transition dipole moments are directed either in the lattice plane (anisotropic case) or perpendicular to the plane (isotropic case). We obtain a general criterion for the modulational instability of a plane wave, which we apply for these two particular cases. In Sec. III we discuss the localized stationary states of the system. We calculate them numerically and use a variational approach to gain some analytical insight into their structure. Also, we discuss their linear stability properties. In Sec. IV we investigate the dynamics of the system with initial conditions chosen either of Gaussian form or as slightly perturbed stationary states. Finally, we summarize our results in Sec. V.

\section{SYSTEM AND EQUATIONS OF MOTION}

We consider a quadratic two-dimensional lattice with lattice spacing equal to 1 . The model we study is described by the Hamiltonian

$$
H=T+U,
$$

where

$$
T=\sum_{\vec{n}, \vec{n}^{\prime}\left(\vec{n}^{\prime} \neq \vec{n}\right)} J_{\vec{n}-\vec{n}^{\prime}} \psi_{\vec{n}}^{*} \psi_{\vec{n}^{\prime}}
$$

is the dispersive energy of the excitation, and

$$
U=\frac{a}{2} \sum_{n}\left|\psi_{n}\right|^{4}
$$

is its potential energy that describes a self-interaction of the quasiparticle ( $a$ is the nonlinearity parameter). In Eqs. (1)(3) $\vec{n}=(m, n, 0)(m, n=0, \pm 1, \pm 2, \ldots)$ is the lattice vector (we assume that the lattice is in the $x-y$ plane), and $\psi_{n}^{-} \equiv \psi_{m, n}$ is the excitation wave function. We will investigate excitations whose dispersion is due to the dipole-dipole interaction

$$
J_{\vec{n}-\vec{n}^{\prime}}=\frac{1}{\left|\vec{n}-\vec{n}^{\prime}\right|^{3}}\left[1-3\left(\vec{d} \cdot \frac{\left(\vec{n}-\vec{n}^{\prime}\right)}{\left|\vec{n}-\vec{n}^{\prime}\right|}\right)^{2}\right],
$$

where $\vec{d}=(\cos \phi \sin \theta, \sin \phi \sin \theta, \cos \theta)$ is the transition dipole moment (its length is normalized to unity, $\theta$ is the colatitude and $\phi$ is the longitude).

From the Hamiltonian (1)-(3) we obtain the equation of motion $i \dot{\psi}_{n}^{-}=\partial H / \partial \psi_{n}^{*}$ for the excitation wave function $\psi_{n}^{\vec{n}}$ in the form

$$
i \dot{\psi}_{n}^{\vec{n}}-\sum_{\vec{n}^{\prime}\left(\vec{n}^{\prime} \neq \vec{n}\right)} J_{\vec{n}-\vec{n}^{\prime}} \psi_{n^{\prime}}-a\left|\psi_{n}\right|^{2} \psi_{n}^{\vec{n}}=0,
$$

where the overdot denotes the time derivative. The Hamiltonian $H(1)-(3)$ and the excitation number

$$
N=\sum_{\vec{n}}\left|\psi_{n}\right|^{2}
$$

are conserved quantities. Obviously, the Lagrangian for Eq. (5) can be written via the Legendre transform of $H$ as

$$
L=i \sum_{n} \frac{1}{2}\left(\dot{\psi}_{n}^{-} \psi_{n}^{*}-\text { c.c. }\right)-H .
$$

Equation (5) has an exact plane-wave solution

$$
\psi_{n}(t)=A e^{i \vec{k} \cdot \vec{n}-i \omega t},
$$

with amplitude $A$ and the frequency $\omega$ being of the form

$$
\omega=a A^{2}+J(\vec{k}),
$$

where the function

$$
J(\vec{k}) \equiv J(k, q)=\sum_{\vec{n}(\vec{n} \neq 0)} J_{\vec{n}} e^{i \vec{k} \cdot \vec{n}}
$$

determines the linear dispersion of the excitations.

To investigate the linear stability of the plane-wave solution determined by Eqs. (8)-(10), we will seek the solution to Eq. (5) in the form

$$
\psi_{\vec{n}}(t)=\left[A+f_{\vec{n}}(t)\right] e^{i \vec{k} \cdot \vec{n}-i \omega t+i v_{n}^{\vec{n}}(t)} .
$$

Inserting Eq. (11) into Eq. (5) and linearizing it with respect to $f_{\vec{n}}$ and $v_{n} \vec{n}$ we get

$$
\begin{aligned}
& \dot{f}_{\vec{n}}=-\sum_{\vec{n}^{\prime}\left(\vec{n}^{\prime} \neq \vec{n}\right)} J_{\vec{n}-\vec{n}^{\prime}} \sin \left[\vec{k} \cdot\left(\vec{n}-\vec{n}^{\prime}\right)\right] f_{\vec{n}^{\prime}} \\
& -A \sum_{\vec{n}^{\prime}\left(\vec{n}^{\prime} \neq \vec{n}\right)} J_{\vec{n}-\vec{n}^{\prime}} \cos \left[\vec{k} \cdot\left(\vec{n}-\vec{n}^{\prime}\right)\right]\left(v_{\vec{n}}-v_{\vec{n}^{\prime}}\right), \\
& A \dot{v_{n}}=\left[J(\vec{k})-2 a A^{2}\right] f_{\vec{n}}-\sum_{\vec{n}^{\prime}\left(\vec{n}^{\prime} \neq \vec{n}\right)} J_{\vec{n}-\vec{n}^{\prime}} \cos \left[\vec{k} \cdot\left(\vec{n}-\vec{n}^{\prime}\right)\right] f_{\vec{n}^{\prime}} \\
& +A \sum_{\vec{n}^{\prime}\left(\vec{n}^{\prime} \neq \vec{n}\right)} J_{\vec{n}-\vec{n}^{\prime}} \sin \left[\vec{k} \cdot\left(\vec{n}-\vec{n}^{\prime}\right)\right]\left(v_{\vec{n}}-v_{\vec{n}^{\prime}}\right) .
\end{aligned}
$$


Looking for the solution of the set of Eqs. (12) in the form

$$
f_{\vec{n}}(t)=F e^{\sigma t+i \vec{\kappa} \cdot \vec{n}}, \quad v_{n}(t)=\Upsilon e^{\sigma t+i \vec{\kappa} \cdot \vec{n}},
$$

where $F$ and $Y$ are the amplitudes, $\sigma$ is the increment, and $\vec{\kappa}$ is the wave number of the modulation wave, we obtain that the increment is given by

$$
\begin{aligned}
(\sigma- & \left.\frac{i}{2}[J(\vec{k}-\vec{\kappa})-J(\vec{k}+\vec{\kappa})]\right)^{2} \\
= & \left(\frac{2 J(\vec{k})-J(\vec{k}-\vec{\kappa})-J(\vec{k}+\vec{\kappa})}{2}\right) \\
& \times\left(2 a A^{2}+\frac{J(\vec{k}-\vec{\kappa})+J(\vec{k}+\vec{\kappa})}{2}-J(\vec{k})\right) .
\end{aligned}
$$

We remark that this expression for the increment $\sigma$ is valid for arbitrary interaction described by $J_{\vec{n}-\vec{n}^{\prime}}$, and it reduces to the expression obtained in Ref. 34 for the one-dimensional case with only nearest-neighbor interactions. Modulational instability will occur when $\sigma$ has a strictly positive real part. In the limit of long-wavelength perturbations, i.e., when $|\vec{\kappa}| \ll 1$, we obtain from Eq. (14),

$$
\left(\sigma+i \frac{\partial J(\vec{k})}{\partial k_{\mu}} \kappa_{\mu}\right)^{2}=-a A^{2} \frac{\partial^{2} J(\vec{k})}{\partial k_{\mu} \partial k_{\nu}} \kappa_{\mu} \kappa_{\nu},
$$

where the summation indices $\mu$ and $\nu$ run over the coordinates $x$ and $y$, and the summation convention is used.

From Eq. (15) it is seen that the necessary condition for modulational instability to occur is positive definiteness of the left-hand side of Eq. (15). For the waves (8)-(10) that correspond to the extrema of the dispersion surface (10) $[\partial J(\vec{k}) / \partial \vec{k}=0]$, the necessary condition is thus positive (negative) definiteness of the effective mass tensor $\partial^{2} J(\vec{k}) / \partial k_{\mu} \partial k_{\nu}$ when self-interaction is attractive $(a<0)$ [repulsive $(a>0)]$.

Let us consider the shape of the dispersion surface given by Eq. (10). We will distinguish two particular cases: (i) the anisotropic case where the dipole moments are in the $x-y$ plane $(\theta=\pi / 2)$ and are parallel to the $x$-axis $(\phi=0)$; (ii) the isotropic case where the dipole moments are perpendicular to the $x-y$ plane $(\theta=0)$.

For the anisotropic case (i), the shape of the dispersion function $J(k, q)$ is shown in Fig. 1(a). It is seen that the function has a minimum at $k=0, q= \pm \pi$ and a maximum at $k= \pm \pi, q=0$. In the Appendix we show that in the vicinity of the minimum $k=0, q=\pi$

$$
J(k, q) \simeq-5.1+1.79 k^{2}+0.15(\pi-q)^{2},
$$

and in the vicinity of the maximum $k=\pi, q=0$

$$
J(k, q) \simeq 6.03-1.17(\pi-k)^{2}-1.29 q^{2} .
$$

The point $k=q=0$ is a saddle point, and near this point we obtain from Eqs. (A19) and (A20) that

$$
J(k, q) \simeq-4.51+2 \pi \frac{k^{2}}{\sqrt{k^{2}+q^{2}}} .
$$

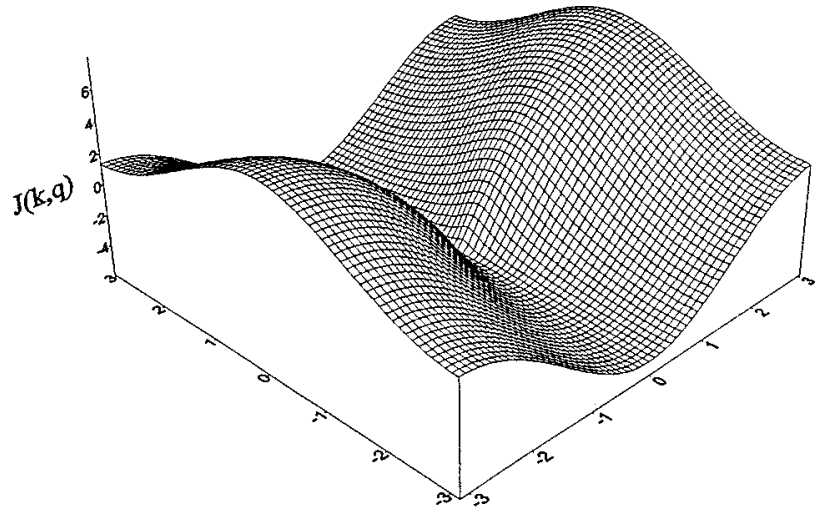

(a)
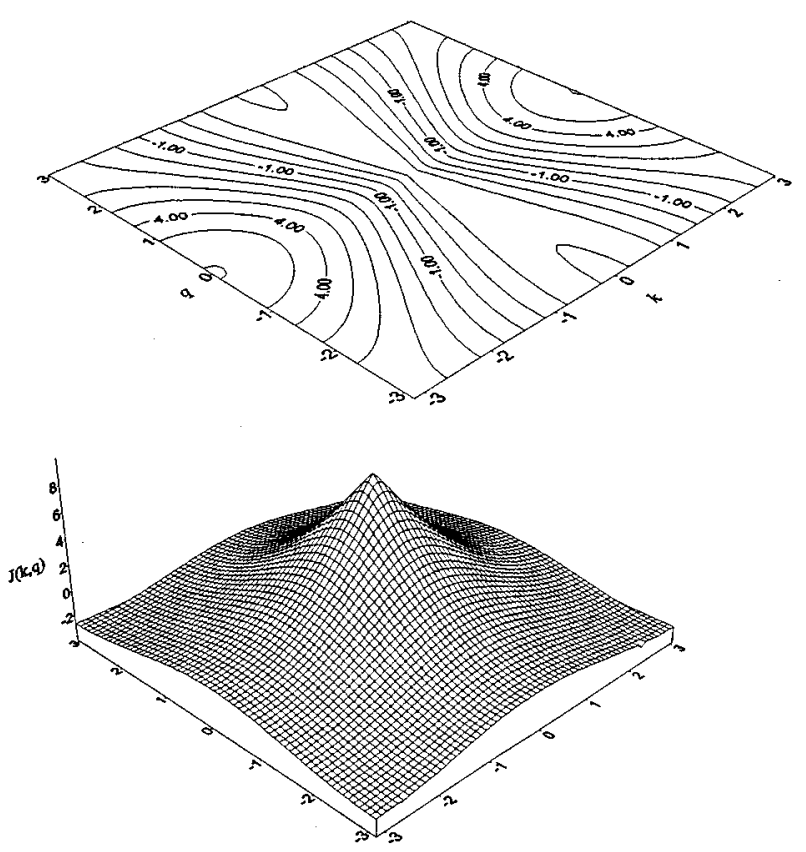

(b)

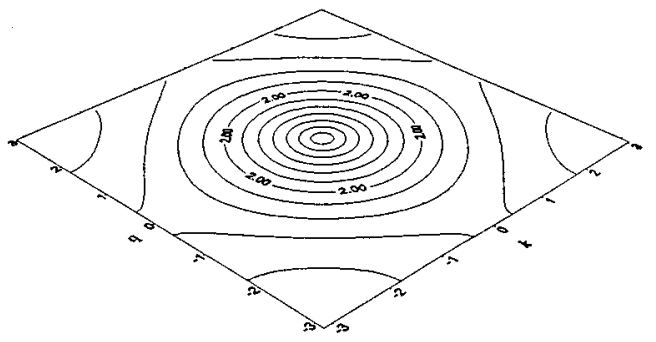

FIG. 1. Linear spectrum $J(k, q)(10)$ of the dispersion operator $J_{\vec{n}}$ for the dipole-dipole interaction (4); (a) anisotropic case $[\vec{d}$ $=(1,0,0)]$; (b) isotropic case $[\vec{d}=(0,0,1)]$.

It is worth noticing that near the maximum the dispersion (17) is almost isotropic, while in the vicinity of the minimum it is highly anisotropic; in the latter case the ratio of the effective masses in the $n$ and $m$ directions exceeds 10 [see Eq. (16)].

The shape of the dispersion surface in the isotropic case (ii) is presented in Fig. 1(b). At the point $k=q=\pi$ the dispersion function has a minimum and has the form (see Appendix) 


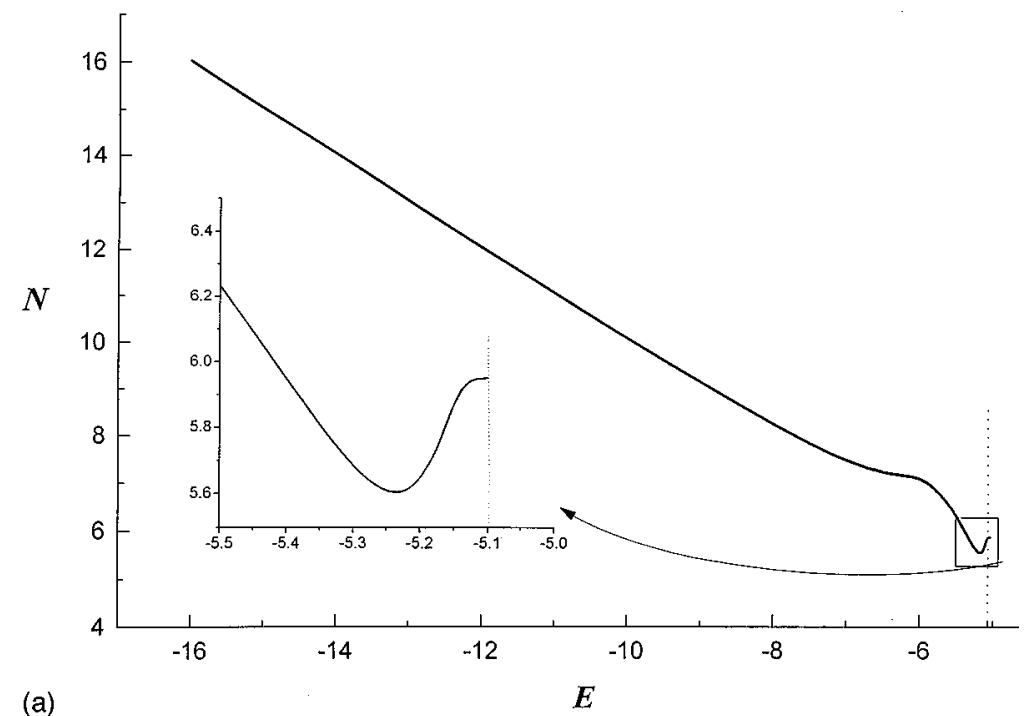

(a)

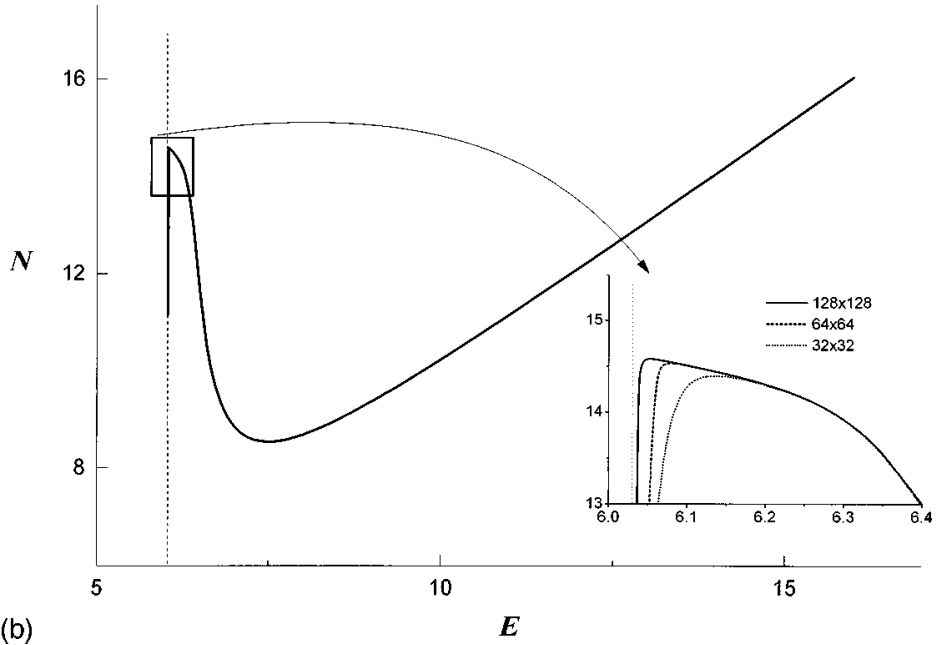

FIG. 2. Excitation number $N$ versus nonlinear frequency $E$ for numerically obtained solitary stationary solutions of the form (21); (a), (b) anisotropic case $[\vec{d}=(1,0,0)]$; (c), (d) isotropic case $[\vec{d}=(0,0,1)]$. In (a) and (c) the nonlinear interaction is attractive $(a=-1)$; in (b) and (d) the interaction is repulsive $(a=+1)$. Insets show detailed behavior near the cutoff frequencies obtained from Eqs. (25) and (26); inset in (b) shows the behavior when the number of sites (indicated in the figure) is increased.

$$
J(k, q) \simeq-2.65+0.4\left[(\pi-k)^{2}+(\pi-q)^{2}\right] .
$$

In close proximity of the point $k=q=0$ the dispersion surface has a peaklike shape, and is described by the function

$$
J(k, q) \simeq 9.03-2 \pi \sqrt{k^{2}+q^{2}} .
$$

In accordance with the modulational instability criterion obtained from Eq. (15), one can conclude that in the anisotropic case the waves (8)-(10) with $k=0, q= \pm \pi$ are modulationally unstable for long-wavelength perturbations when the self-interaction (3) is attractive $(a<0)$, and the waves $k= \pm \pi, q=0$ are unstable for the repulsive self-interaction $(a>0)$. In the isotropic case the modulational instability occurs for the waves with $k= \pm \pi, q= \pm \pi$ when $a<0$, and for the wave $k=q=0$ when $a>0$. [In the latter case, this is seen by direct substitution of Eq. (20) into Eq. (14).]

\section{STATIONARY STATES OF THE SYSTEM}

We are interested here in the stationary solutions of Eq. (5) of the form

$$
\psi_{\vec{n}}(t)=\Psi_{\vec{n}} e^{-i E t}
$$

with a real shape function $\Psi_{\vec{n}} \equiv \Psi_{m, n}$ and a nonlinear frequency $E$. The equation of motion (5) then yields the governing equation for the functions $\Psi_{m, n}$ as

$$
E \Psi_{m, n}=\sum_{m^{\prime}, n^{\prime}} J_{m^{\prime}, n^{\prime}} \Psi_{m-m^{\prime}, n-n^{\prime}}+a \Psi_{m, n}^{3}
$$

As was shown above, modulational instability may occur for linear waves (8) with wave vectors belonging to the boundaries or the center of the first Brillouin zone of the square lattice. In the cases when the instability occurs at the Brillouin-zone boundaries, it is natural to expect that the nonlinear excitations that appear as a result of this instability will be staggered. Thus, the corresponding wave function $\Psi_{m, n}$ can be written in the form

$$
\Psi_{m, n}=e^{i(K m+Q n)} \Phi_{m, n},
$$

where the envelope function $\Phi_{m, n}$ satisfies the equation 


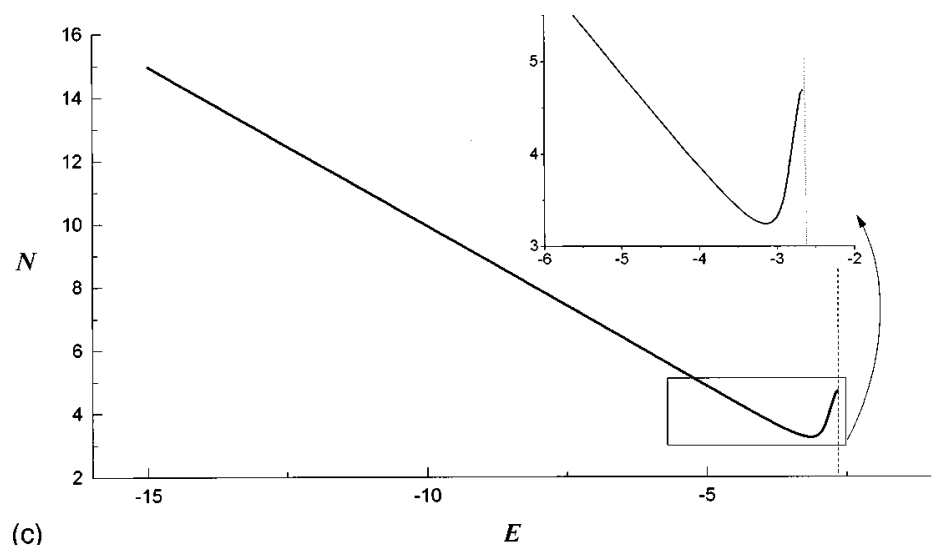

(c)

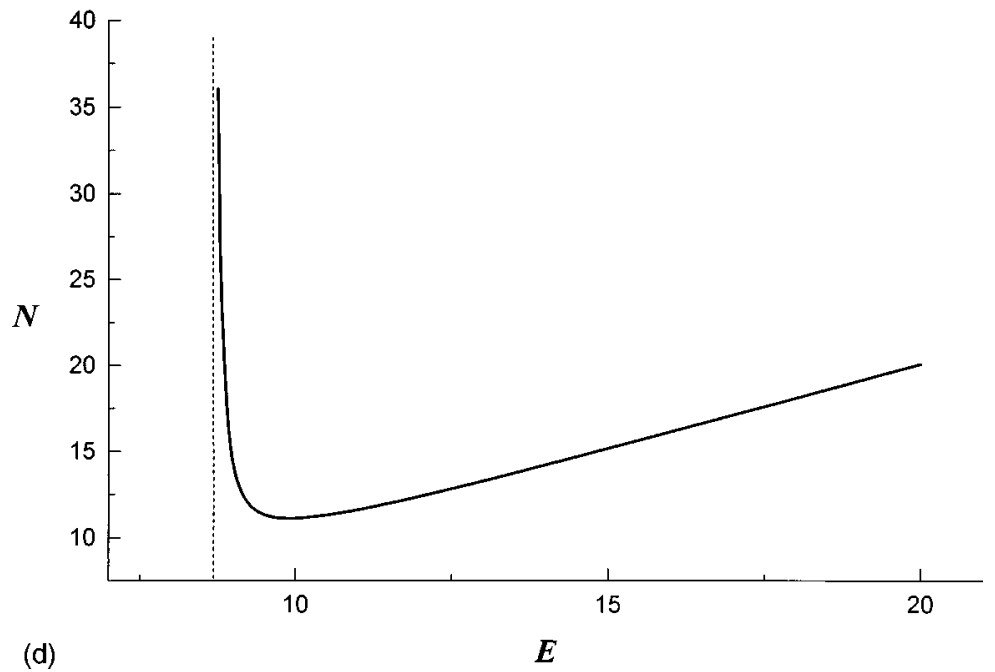

FIG. 2. (Continued).

$$
E \Phi_{m, n}=\sum_{m^{\prime}, n^{\prime}} J_{m^{\prime}, n^{\prime}} e^{i\left(K m^{\prime}+Q n^{\prime}\right)} \Phi_{m-m^{\prime}, n-n^{\prime}}+a \Phi_{m, n}^{3} .
$$

The vector $(K, Q)$ corresponds to one of the boundaries $[(K, Q)=(0, \pm \pi),( \pm \pi, 0),( \pm \pi, \pm \pi)]$ or the center $[(K, Q)=(0,0)]$ of the first Brillouin zone. In the latter case, the function (23) describes an unstaggered excitation.

We have studied the nonlinear eigenvalue problem given by Eq. (22) numerically and analytically. The numerical procedure used is a discrete version of the iterative Petviashvili method described in Ref. 35. Throughout, zero boundary conditions were used, and the maximum residual error of the numerical solution $\Psi_{m, n}$ of Eq. (22) never exceeded $10^{-10}$.

Figures 2(a) -2 (d) show the dependence $N(E)$, where $N$ is the excitation number (6), for the solitary states (21) obtained numerically as solutions of Eq. (22) for the in-plane [Figs. 2(a) and 2(b)] and out-of-plane [Figures 2(c) and 2(d)] alignments of the dipole moments. Figures 2 (a) and 2(c) correspond to the attractive nonlinear interaction $(a=-1)$. Here the localized states have frequencies lying below the linear dispersion surface,

$$
\begin{gathered}
E \leqslant J(0, \pi) \simeq-5.1 \text { when } \vec{d}=(1,0,0) \\
E \leqslant J(\pi, \pi) \simeq-2.65 \text { when } \vec{d}=(0,0,1) .
\end{gathered}
$$

In Figs. 2(b) and 2(d) the nonlinear interaction (3) is repulsive $(a=1)$, and the eigenfrequencies lie above the upper edge of the dispersion surface,

$$
\begin{aligned}
& E \geqslant J(\pi, 0) \simeq 6.03 \text { when } \vec{d}=(1,0,0) ; \\
& E \geqslant J(0,0) \simeq 9.03 \text { when } \vec{d}=(0,0,1) .
\end{aligned}
$$

Figures 3-5 show some examples of these solutions. It is seen that, at least when the excitations are relatively wide, their shapes vary in accordance with Eq. (23): while in the anisotropic case (in-plane dipole alignment) the eigenfunctions are modulated either along the $m$ or $n$ directions (Figs. 3 and 4), in the isotropic case (dipole moments perpendicular to the plane) the functions are either modulated along both the $m$ and $n$ directions $(a<0)$ or they are nonmodulated $(a>0)$ (Fig. 5). However, we note that for narrow excitations, the states may be staggered only in a neighborhood around its central peak, and unstaggered outside this region [see, e.g., the contour plots in Figs. 3(a), 4(b), and 4(c)]. The existence of this type of solutions is a consequence of the long-range nature of the dispersion; in the nearestneighbor case all solitary stationary states are either completely staggered or unstaggered as a consequence of the symmetric nature of the dispersion surface. [The nearestneighbor equation is invariant under the simultaneous transformations $\Psi_{m, n} \rightarrow(-1)^{\mu m+\nu n} \Psi_{m, n}, \quad J_{ \pm 1,0} \rightarrow(-1)^{\mu} J_{ \pm 1,0}$, 


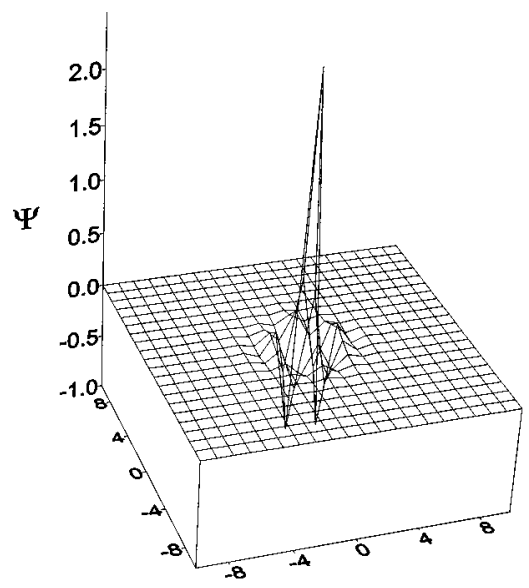

(a)
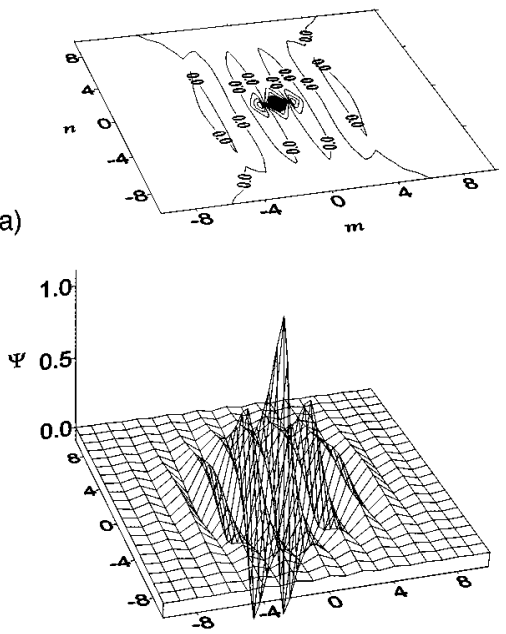

(b)

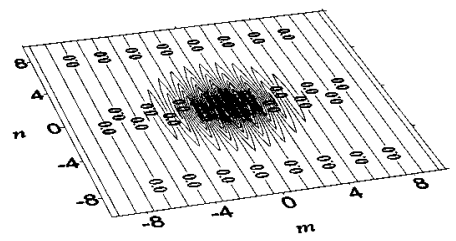

FIG. 3. Shape functions $\Psi_{m, n}$ with contour plots for stationary solutions (21) in the case of anisotropic dispersion $[\vec{d}=(1,0,0)]$ and repulsive nonlinearity $(a=1)$; (a) linearly stable solution for $E$ $=8$; (b) unstable solution for $E=6.3$.

$J_{0, \pm 1} \rightarrow(-1)^{\nu} J_{0, \pm 1}$, while no such invariance exists for the general case of long-range dispersion.]

In Fig. 2(b) the dependence of $N$ on the grid size is presented. When the number of sites increases the influence of the boundaries becomes smaller, and this is reflected in the narrowing of the dip in the region of $E$ close to the threshold value $[J(\pi, 0)$ in this case $]$. It is seen that the level of the flat plateau around the local maximum near $J(\pi, 0)$ approaches the asymptotic value of the excitation number $N$ corresponding to the ground state of the continuum NLS equation, which can be obtained from Eq. (24) for the envelope function $\Phi_{m, n}$. Indeed, taking into account the dispersion law given by Eq. (17), we obtain that the continuum limit of Eq. (24) near the threshold value $J(\pi, 0)$ for $a=1$ has the form

$$
\left(1.17 \frac{\partial^{2}}{\partial m^{2}}+1.29 \frac{\partial^{2}}{\partial n^{2}}\right) \Phi+\Phi^{3}-[E-J(\pi, 0)] \Phi=0 .
$$

It is well known ${ }^{36}$ that the ground-state solution of the isotropic two-dimensional NLS equation

$$
\left(\frac{\partial^{2}}{\partial x^{2}}+\frac{\partial^{2}}{\partial y^{2}}\right) \phi+\phi^{3}+\Lambda \phi=0
$$

exists for $\int_{-\infty}^{\infty} d x \int_{-\infty}^{\infty} d y \phi^{2} \simeq 11.7$. Rescaling the spatial variables $m$ and $n$ in Eq. (27), we obtain that the threshold value of the excitation number for the excitations (23) with $K$ $=\pi, Q=0$ is 14.4 , which is in fair agreement with the results presented in Fig. 2(b). The same dependence of the shape of the function $N(E)$ on the grid size takes place for all cases shown in Fig. 2, except for the case of a repulsive nonlinearity in the lattice with out-of-plane dipole moments [Fig. $2(\mathrm{~d})$ ]. Here $N(E)$ monotonically increases when $E \rightarrow J(0,0)$. This behavior can also be explained by taking into account the peculiarities of the linear dispersion in the isotropic case. Indeed, it is seen from Eq. (20) that for $a=1$ and $E$ close to the top of the dispersion surface $k=q=0$, the continuum limit of Eq. (24) has the form

$$
-\sqrt{-\nabla^{2}} \Phi+\Phi^{3}-[E-J(0,0)] \Phi=0,
$$

where

$$
\sqrt{-\nabla^{2}} \equiv\left(-\frac{\partial^{2}}{\partial m^{2}}-\frac{\partial^{2}}{\partial n^{2}}\right)^{1 / 2}
$$

is the Fourier multiplier operator defined by

$$
\begin{aligned}
& \int_{-\infty}^{\infty} d m \int_{-\infty}^{\infty} d n e^{i(k m+q n)} \sqrt{-\nabla^{2}} \Phi(m, n) \\
& =\sqrt{k^{2}+q^{2}} \int_{-\infty}^{\infty} d m \int_{-\infty}^{\infty} d n e^{i(k m+q n)} \Phi(m, n) .
\end{aligned}
$$

The behavior close to the threshold $J(0,0)$ can easily be understood, since the scaling transformation $\Phi=[E$ $-J(0,0)]^{1 / 2} R(x, y), x=[E-J(0,0)] m, y=[E-J(0,0)] n$ reduces Eq. (29) to

$$
-\left(-\frac{\partial^{2}}{\partial x^{2}}-\frac{\partial^{2}}{\partial y^{2}}\right)^{1 / 2} R+R^{3}-R=0
$$

which is independent of $E$. The applied scaling therefore yields

$$
N=\int_{-\infty}^{\infty} d m \int_{-\infty}^{\infty} d n \Phi(m, n) \sim[E-J(0,0)]^{-1},
$$

which agrees with the results of the numerical simulations [see Fig. 2(d)]. It is interesting to note that an equation of the same type as Eq. (29) (but with a quadratic instead of cubic nonlinearity) arises in the theory of low-frequency oscillations of a boundary layer with high Reynolds number ${ }^{37}$ (see also Ref. 38). For this equation, it was shown ${ }^{38}$ that onedimensional solitons are unstable with respect to twodimensional perturbations. 

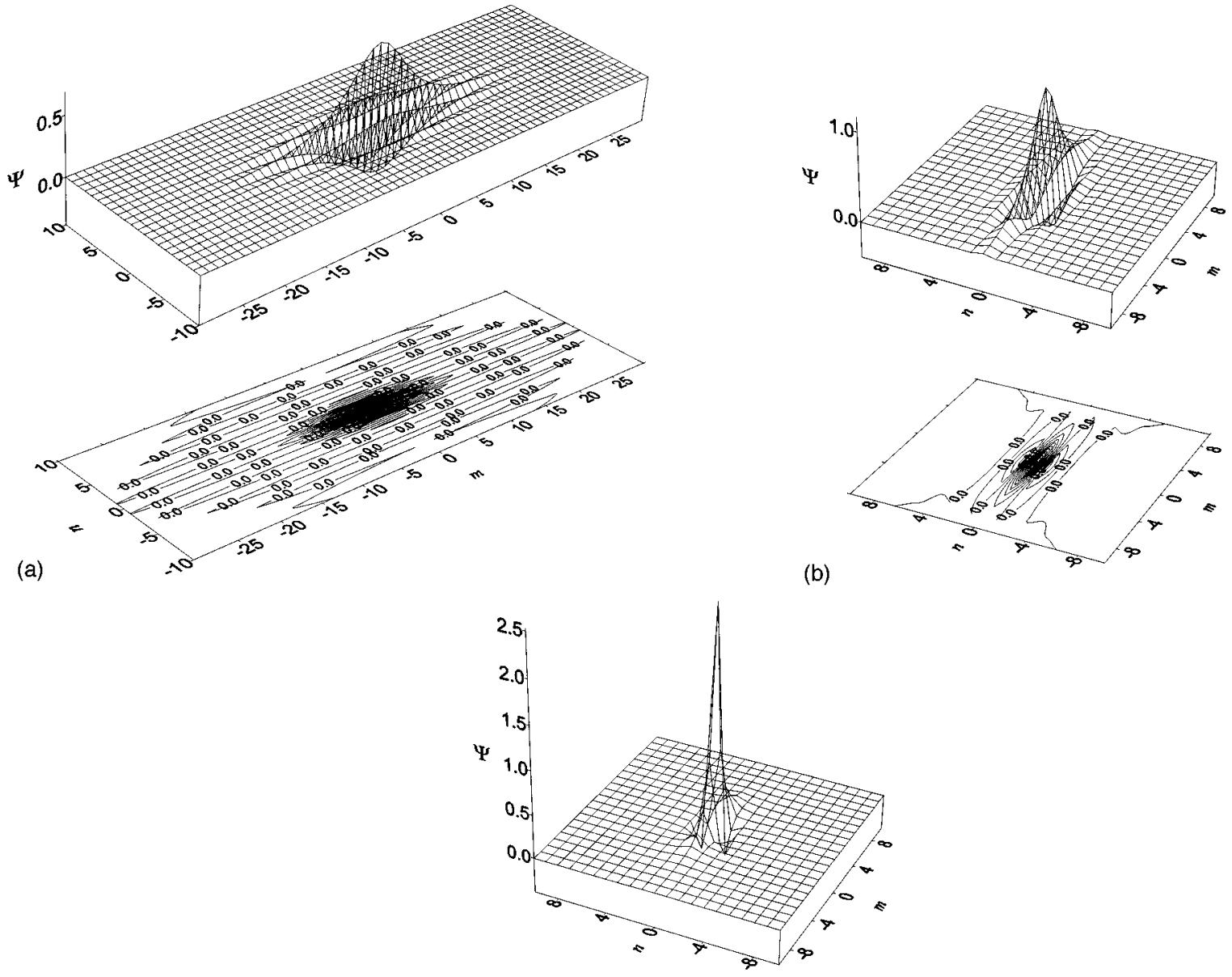

(b)

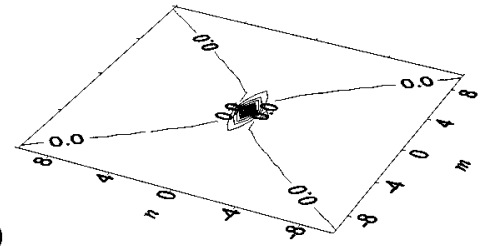

(c)

FIG. 4. Same as Fig. 3 but for attractive nonlinearity $(a=-1)$; (a) unstable solution for $E=-5.2$; (b), (c) stable solutions for (b) $E$ $=-5.5$ and (c) $E=-8$.

Recently, a criterion for instability of solitary excitations in a discrete two-dimensional NLS model with nearestneighbor dispersive interaction was obtained. ${ }^{39}$ It was shown that the stationary states are unstable for

$$
\frac{\partial N}{\partial|E|}<0
$$

Using the approach proposed in Ref. 39, it is easy to show that the criterion (34) is valid also in the case of long-range dispersion. From this criterion, we conclude from the shape of the curves $N(E)$ in Fig. 2 that, similarly to the case with only nearest-neighbor interaction, ${ }^{39}$ the broad stationary states are generally unstable [examples of unstable states are shown in Figs. 3(b) and 4(a)]. For the one-dimensional case with nearest-neighbor ${ }^{40}$ or long-range ${ }^{30}$ dispersion, it has been shown that localized stationary states, whose envelope has a single maximum at a lattice site (on-site states) and for which

$$
\frac{\partial N}{\partial|E|}>0
$$

are linearly stable. Although we have not been able to rigorously extend this criterion to the two-dimensional case, we believe that also for the cases considered here, the condition (35) is a necessary and sufficient condition for linear stability of on-site localized stationary states (a similar assumption was employed in Refs. 20 and 41 for the case of nearestneighbor dispersion). As will be illustrated in Sec. IV, this conjecture, which implies, e.g., that the states shown in Figs. $3(\mathrm{a}), 4(\mathrm{~b})$ and 4(c), and 5 are linearly stable, is also supported by numerical dynamical simulations.

It is worth noting that in the case of an attractive nonlinear interaction and in-plane dipole alignment, the dependence $N(E)$ reveals in the interval of stability $[(\partial N / \partial|E|)$ $>0$ ] the existence of two different slopes $\partial N / \partial|E|$ [see Fig. 2(a)]. Figure 4 shows that the shapes of the corresponding solutions differ significantly. The states with low frequency 


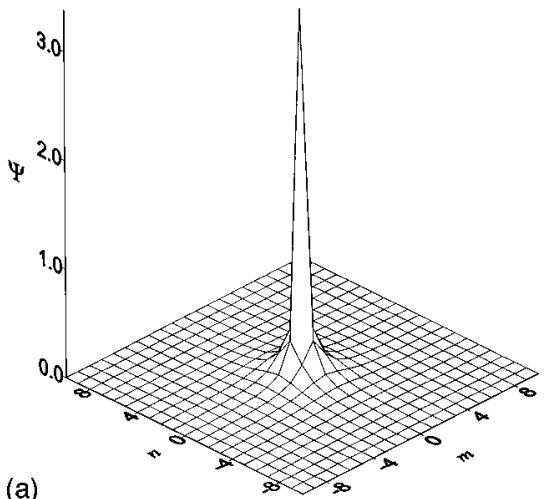

(a)

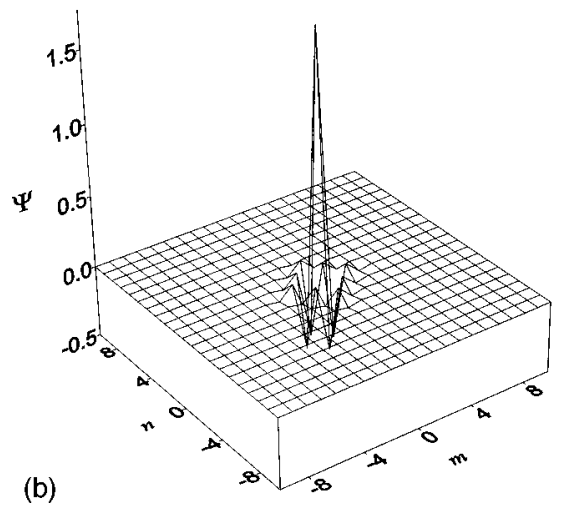

FIG. 5. Same as Figs. 3 and 4 but for isotropic dispersion $[\vec{d}$ $=(0,0,1)]$ and (a) repulsive nonlinearity $(a=1)$ and $E=12$; (b) attractive nonlinearity $(a=-1)$ and $E=-4.0$. In both cases, the solution is found to be linearly stable.

$|E|$ have an ellipselike projection on the lattice plane. The major axis of the ellipse is parallel to the $m$ axis and its eccentricity is close to one [see Fig. 4(b)]. In contrast, the solutions with high frequency $|E|$ represent almost isotropic intrinsically localized states with a width of a few lattice constants in both directions [see Fig. 4(c)]. It is clear that the reason for this difference is the anisotropy of the linear dispersion given by Eq. (16) [in the cases where the linear dispersion is (almost) isotropic, all solutions are (almost) isotropic as illustrated in Figs. 3 and 5], but to gain insight into this phenomenon we need to have a solution of the problem which is valid in the whole interval of the nonlinear frequencies.

To obtain the needed solution of the problem we will use a variational approach. As an ansatz for a localized state we choose

$$
\Phi_{m, n}=\sqrt{N} f_{m}(\alpha) f_{n}(\beta),
$$

where

$$
f_{j}(z)=\sqrt{\tanh z} e^{-z|j|}, \quad z=\alpha, \beta,
$$

with $\alpha$ and $\beta$ being trial parameters. It is seen from Eqs. (36) and (37) that $\alpha^{-1}\left(\beta^{-1}\right)$ is the excitation width in the $m(n)$ direction. The functions (36) and (37) automatically satisfy the normalization condition

$$
\sum_{m, n} \Psi_{m, n}^{2}=N
$$

so that the problem of minimizing $H$ under the condition $N$ $=$ const is reduced to the problem of satisfying the equations

$$
\frac{\partial H}{\partial \alpha}=0, \frac{\partial H}{\partial \beta}=0 .
$$

To calculate the kinetic energy $T$ we use the discrete Fourier transformation

$$
\bar{\Phi}(k, q)=\sum_{m, n} e^{i(k m+q n)} \Phi_{m, n}=\sqrt{N} \bar{f}(k, \alpha) \bar{f}(q, \beta),
$$

where

$$
\bar{f}(k, \alpha)=\sqrt{\tanh \alpha} \frac{\sinh \alpha}{\cosh \alpha-\cos k} .
$$

This permits us to rewrite Eq. (2) as

$$
\begin{aligned}
T(K, Q)= & \frac{1}{M} \sum_{k, q} J(K+k, Q+q)[\bar{\Phi}(k, q)]^{2} \\
= & \frac{N}{4 \pi^{2}} \int_{0}^{2 \pi} d k d q J(K+k, Q+q) \\
& \times[\bar{f}(k, \alpha)]^{2}[\bar{f}(q, \beta)]^{2},
\end{aligned}
$$

where $M$ is the number of sites in the system $(M \rightarrow \infty)$. Taking into account the definition (10) of the dispersion function $J(k, q)$, we obtain that the kinetic energy of the system can be represented in the form

$$
T(K, Q)=2 N F(K, Q),
$$

with

$$
\begin{aligned}
F(K, Q)= & \sum_{m=1}^{\infty} \sum_{n=-\infty}^{\infty} J_{m, n} e^{i(K m+Q n)} e^{-(\alpha m+\beta|n|)} \\
& \times(1+m \tanh \alpha)(1+|n| \tanh \beta) \\
& +\sum_{n=1}^{\infty} J_{0, n} e^{i Q n}(1+n \tanh \beta) e^{-\beta n}
\end{aligned}
$$

Inserting Eq. (36) into Eq. (3) we get

$$
U=\frac{a N^{2}}{2} \frac{\tanh ^{2} \alpha}{\tanh (2 \alpha)} \frac{\tanh ^{2} \beta}{\tanh (2 \beta)}
$$

From Eq. (22) we obtain that the nonlinear frequency $E$ can be expressed as

$$
E=\frac{1}{N}[T(K, Q)+2 U],
$$

with $T$ and $U$ being defined by Eqs. (43) and (44).

The two particular types of dipole moment alignments, isotropic case $[\vec{d}=(0,0,1)]$ and anisotropic case $[\vec{d}$ $=(1,0,0)]$, will be considered separately.

\section{A. Out-of-plane dipole alignment}

It is seen from Eq. (4) that when $\vec{d}=(0,0,1)$, the matrix element of the excitation transfer $J_{m, n}$ has the form 


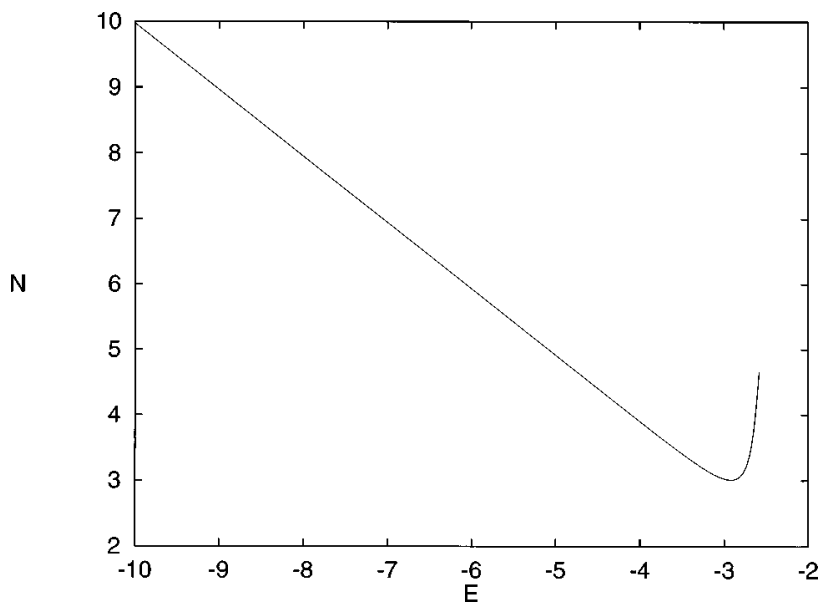

FIG. 6. Excitation number $N$ versus nonlinear frequency $E$ for the isotropic and attractive case considered in Figs. 2(c) and 5(b), obtained from the approximate analytical expression (48).

$$
J_{m, n}=\left(m^{2}+n^{2}\right)^{-3 / 2}
$$

Since $J_{m, n}$ is isotropic, we can restrict ourselves by the isotropic trial functions (36) with $\alpha=\beta$. In the case of attractive nonlinear interaction $(a<0)$, the modulational instability occurs for $K= \pm \pi$ and $Q= \pm \pi$. This means that the nonlinear excitations are staggered both along the $m$ and $n$ directions of the square lattice. Due to phase modulation the series in the right-hand side of Eq. (43b) are rapidly converging for all $\alpha$, and with a good accuracy (of few per cent) they can be approximated by their first terms, yielding

$$
T(\pi, \pi) \simeq N\left(-4 \operatorname{sech} \alpha+\sqrt{2} \operatorname{sech}^{2} \alpha\right) .
$$

The equation $d H / d \alpha=0$, with $H=T(\pi, \pi)+U$, then yields for $a=-1$

$$
N \simeq 8 \frac{\cosh ^{4} \alpha(2 \cosh \alpha-\sqrt{2})}{[2 \cosh (2 \alpha)-1] \cosh (2 \alpha)} .
$$

Figure 6 shows the dependence $N(E)$ obtained analytically from Eqs. (44), (45), (47), and (48). Comparing with Fig. 2(c), it is seen that there is a good qualitative agreement between the analytical and numerical approaches.

\section{B. In-plane dipole alignment}

When the dipole moments are aligned along the $m$ direction $[\vec{d}=(1,0,0)]$, the matrix element of the excitation transfer is

$$
J_{m, n}=\left(1-3 \frac{m^{2}}{m^{2}+n^{2}}\right) \frac{1}{\left(m^{2}+n^{2}\right)^{3 / 2}} .
$$

We consider the case of attractive nonlinear interaction ( $a$ $=-1)$. In this case the nonlinear excitations are modulated along the $n$ direction $(K=0, Q=\pi)$, and the variational equations (39) take the form

$$
\frac{\sinh (4 \alpha)}{2 \cosh (2 \alpha)-1} \frac{\partial F(K, Q)}{\partial \alpha}=\frac{\sinh (4 \beta)}{2 \cosh (2 \beta)-1} \frac{\partial F(K, Q)}{\partial \beta},
$$

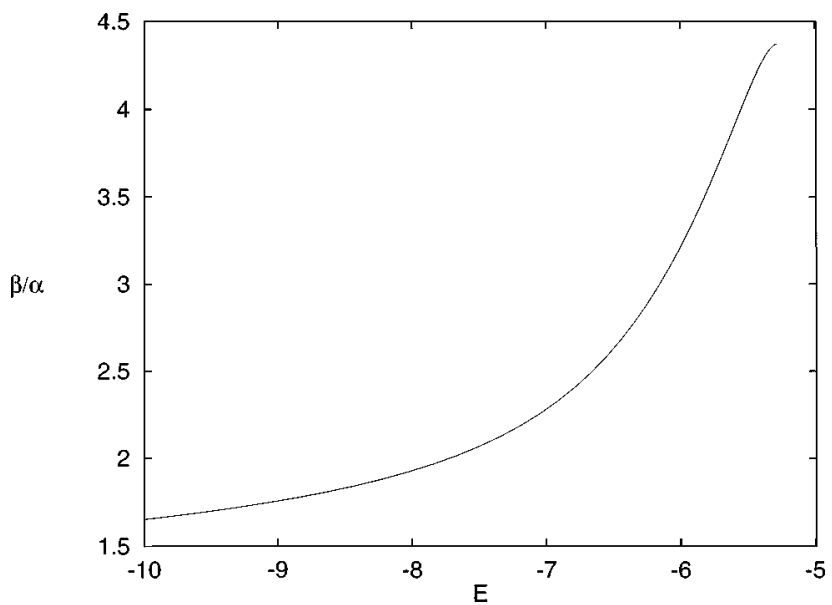

FIG. 7. The dependence on the nonlinear frequency $E$ of the ratio of the excitation widths in the $m$ and $n$ directions $\alpha^{-1}$ and $\beta^{-1}$, as defined by Eqs. (36) and (37), obtained using the approximate expression (50) for the anisotropic and attractive case considered in Figs. 2(a) and 4.

$$
N=8 \frac{\tanh (2 \beta)}{\tanh ^{2} \beta} \frac{\cosh ^{4} \alpha}{2 \cosh (2 \alpha)-1} \frac{\partial F(K, Q)}{\partial \alpha} .
$$

Equation (50), describing the link between the inverse widths $\alpha$ and $\beta$, characterizes the shape of the excitation. Figure 7 shows that in accordance with the results of the numerical simulations represented in Fig. 4, the shape of the excitations change when the absolute value of the nonlinear frequency $|E|$ increases. When the frequency $E$ is close to the edge $J(0, \pi)$ of the linear dispersion band the excitation has an ellipselike shape with an eccentricity $e=\sqrt{1-\alpha^{2} / \beta^{2}} \simeq 0.98$, but for large $|E|$ the excitation is more isotropic with the eccentricity $e \simeq 0.78$. The dependence $N(E)$ obtained from Eqs. (50) and (51) is plotted in Fig. 8. It is seen that the analytical results agree qualitatively with the numerical results obtained from direct solution of Eq. (22) [see Fig. 2(a)]. It is also worth noticing that the characteristic change of the slope of the dependence $N(E)$ occurs in the same energy interval where the shape of the excitation changes. As it was stressed above such a behavior is coupled with the highly

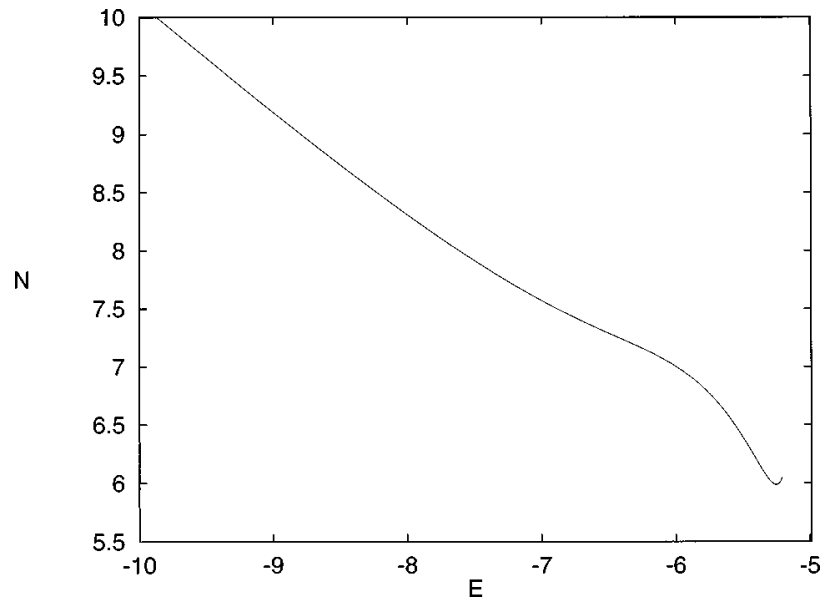

FIG. 8. Excitation number $N$ versus nonlinear frequency $E$ for the anisotropic and attractive case considered in Figs. 2(a), 4, and 7, as obtained from the approximate analytical expression (51). 


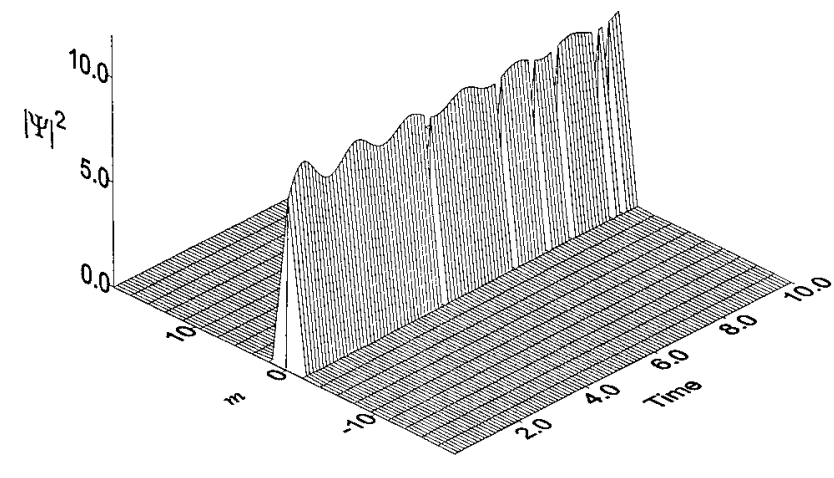

FIG. 9. Dynamical solution of Eq. (5) with anisotropic dipoledipole interaction (4) $[\vec{d}=(1,0,0)]$ and repulsive nonlinearity ( $a$ $=1$ ), where the initial condition is the perturbed stable stationary state (52) with $\epsilon=0.1$ and $\Psi_{m, n}$ as in Fig. 3(a). The figure shows a cut along the axis $n=0$.

anisotropic linear dispersion in the vicinity of the minimum $k=0, q=\pi$ (the ratio of the effective masses in the $n$ and $m$ directions exceeds 10), and as a consequence with the existence of two different characteristic length scales.

\section{DYNAMICAL SIMULATIONS}

In this section, we investigate numerically the dynamics of Eq. (5) with the dipole-dipole dispersive coupling (4) for some different choices of initial conditions. The numerical approach used to integrate the equation is the so-called splitstep Fourier method where the linear and nonlinear parts of the equation are integrated separately (see Ref. 42 for the details). The advantage of this method in this case is that the linear part is solved in Fourier space where the long-range interaction is reduced to a multiplication of the interaction term $J(\vec{k})$ and excitation wave function.

First, we consider the anisotropic case when $\vec{d}=(1,0,0)$ and $J_{m, n}$ is given by Eq. (49). Figures 9 and 10 show the time evolution for the case of repulsive nonlinearity $(a=1)$ with slightly perturbed stationary states of the form (21) as initial conditions:

$$
\psi_{m, n}(0)=(1+\epsilon) \Psi_{m, n},
$$

where $\epsilon=0.1$ and $\Psi_{m, n}$ is the shape function for a stationary state with nonlinear frequency $E$. In Fig. 9, the initial condition is obtained from the stationary state with $E=8$ shown in Fig. 3(a), which corresponds to the linearly stable branch of

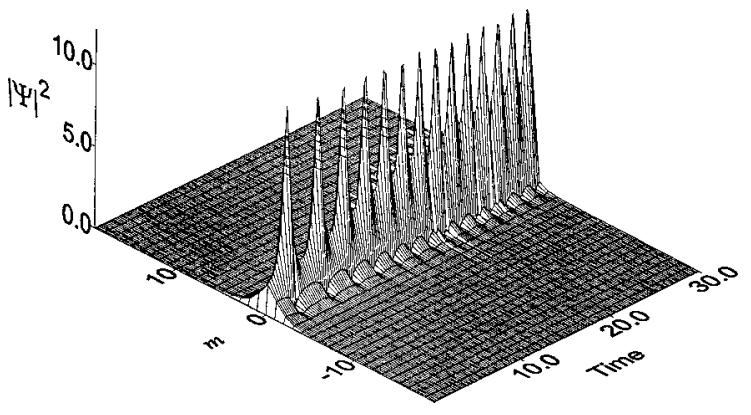

FIG. 10. Same as in Fig. 9, but with the perturbed unstable stationary state with $\Psi_{m, n}$ as in Fig. 3(b) as initial condition.
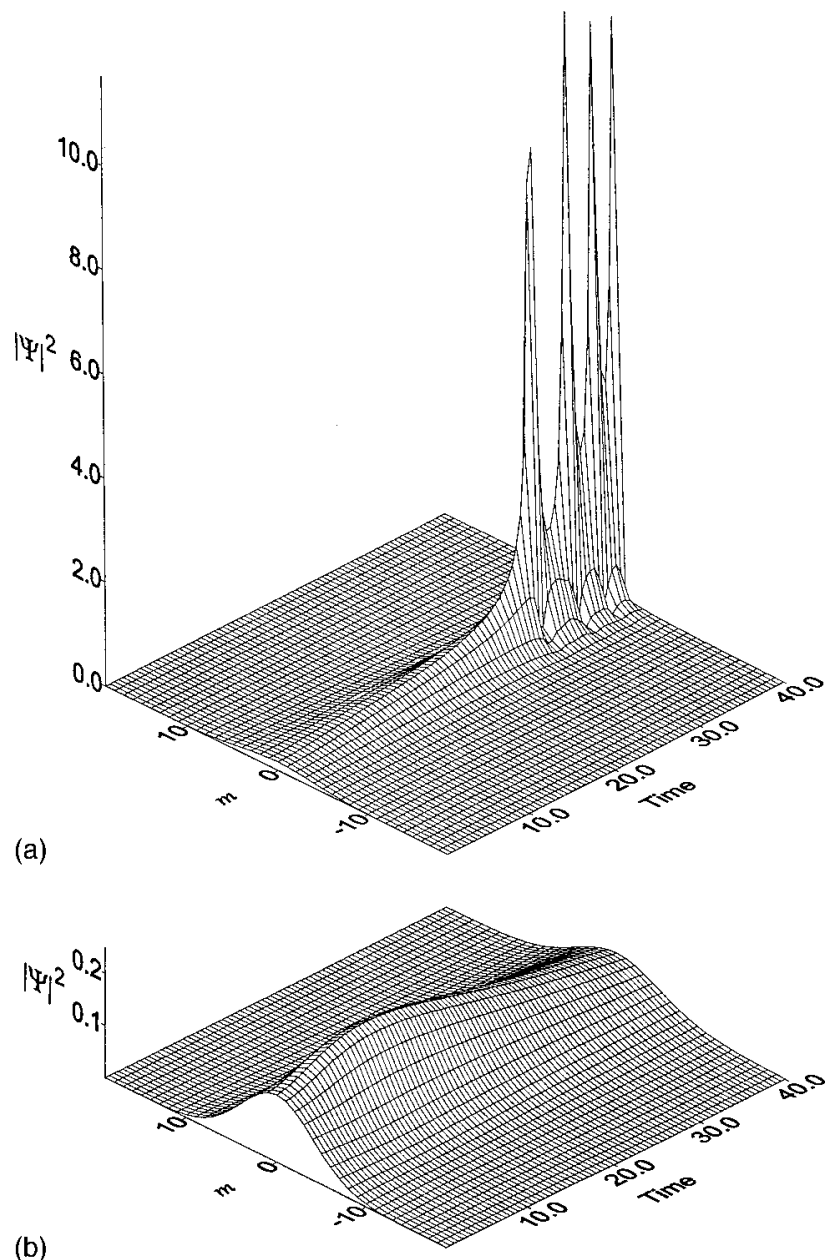

(b)

FIG. 11. Same as in Figs. 9 and 10, but with the Gaussian profile (53) with $K=\pi, Q=0, m_{0}=n_{0}=8$, and (a) $N=15$ ( $A$ $\simeq 0.38)$, resp. (b) $N=13.5(A \simeq 0.36)$, as initial condition.

the curve $N(E)$ in Fig. 2(b) according to the criterion (35). As can be seen, the perturbation causes initially some small oscillations around the initial excitation, but for longer times the solution relaxes into a stationary state of essentially the same form as the initial state, with only a small amount of radiation emitted. On the contrary, when the initial condition is obtained from the stationary state with $E=6.3$ shown in Fig. 3(b), which belongs to the unstable part of the curve $N(E)$ in Fig. 2(b) according to the criterion (34), the time evolution is completely different as shown in Fig. 10. Initially, the rather broad initial excitation collapses into a very narrow state. After this follows a time regime of large but decaying oscillations between narrow and wide states, and finally the system seems to settle down in a narrow state that appears to be a stable stationary state like the one in Fig. 9. Thus, Figs. 9 and 10, together with a number of other similar results obtained for other parameter values, support the conjecture from Sec. III that the criterion (35) is both a necessary and sufficient condition for linear stability of on-site localized stationary states also for the two-dimensional case with long-range dipole-dipole interactions.

In Fig. 11, we show the time evolution for the case of anisotropic dispersion and repulsive nonlinearity of an initial staggered Gaussian profile of the form 

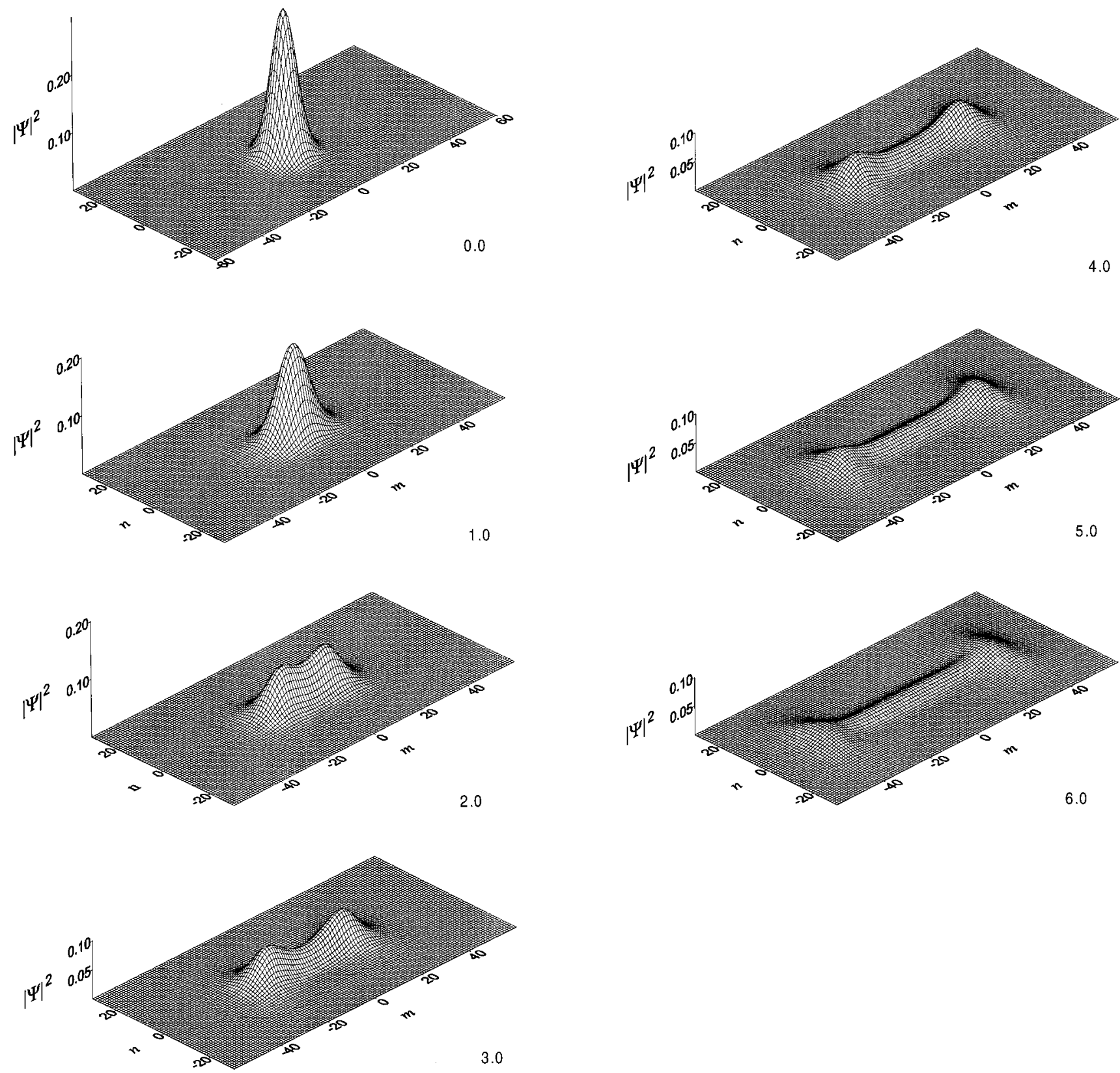

6.0

FIG. 12. Time evolution of an unstaggered initial Gaussian profile (53) with $K=0, Q=0$, with total excitation number $N=25$, for the anisotropic and repulsive case $[\vec{d}=(1,0,0), a=1]$.

$$
\psi_{m, n}(0)=A e^{i(K m+Q n)} \exp \left(-\frac{m^{2}}{m_{0}^{2}}-\frac{n^{2}}{n_{0}^{2}}\right),
$$

with $K=\pi, Q=0$, and $m_{0}=n_{0}=8$, for two different values of the amplitude $A$. In Fig. 11(a) [Fig. 11(b)], the total excitation number is $N=15.0(N=13.5)$. Recalling the result from Sec. III that the continuum limit of the discrete NLS equation in this case can be written in the form (27), whose ground state solution has the excitation number $N=N_{s} \simeq 14.4$, we see that Fig. 11(a) [Fig. 11(b)] corresponds to the case when $N>N_{s}\left(N<N_{s}\right)$. It is seen that the initial excitation with $N>N_{s}$ collapses into an intrinsically localized mode, while the excitation with $N<N_{s}$ disperses. This indicates the existence of a critical threshold for (quasi)collapse of broad initial excitations also for the cases considered here, similarly to what is known $^{36}$ for the ordinary con- tinuum NLS equation. In the case of nearest-neighbor interaction, such a collapse threshold was clearly demonstrated in Ref. 43. Thus, these results indicate that the staggered excitations in the systems with anisotropic dipole-dipole dispersion behave in a similar manner as localized excitations in nearest-neighbor discrete NLS systems.

Figures 12 and 13 show the evolution of two initially Gaussian distributed fields with the same envelope and the same value of the excitation number $(N=25)$, but with different phase configurations. In Fig. 12 the initial distribution is isotropic $(K=Q=0)$, while in Fig. 13 it is staggered in the $m$ direction $(K=\pi)$. It is clearly seen that while the staggered excitation in Fig. 13 collapses just as for the case in Fig. 11(a) (since $N>N_{s}$ ), the unstaggered initial excitation in Fig. 12 splits and spreads. These observations can, in the continuum limit, be related to the behavior of the anisotropic two-dimensional NLS equation 


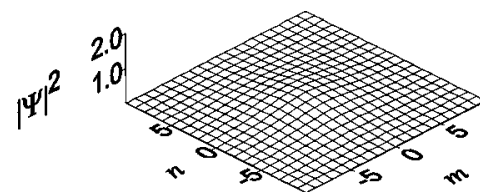

2.0

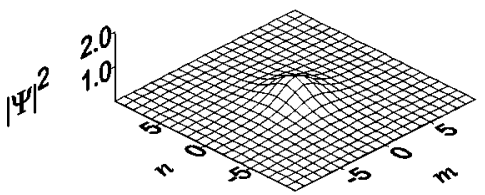

6.0

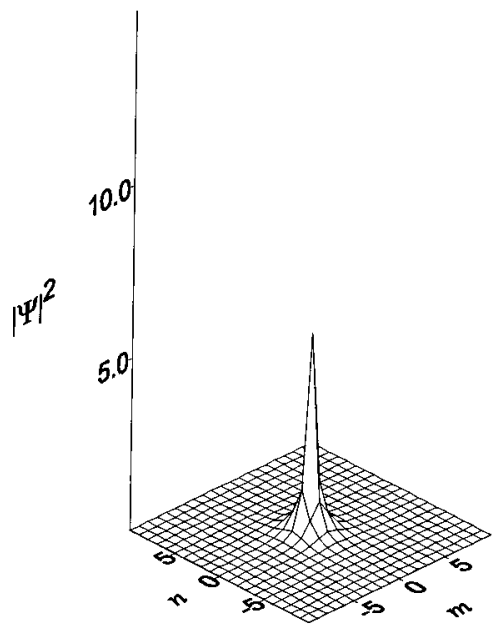

9.0

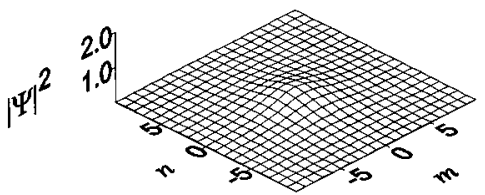

4.0

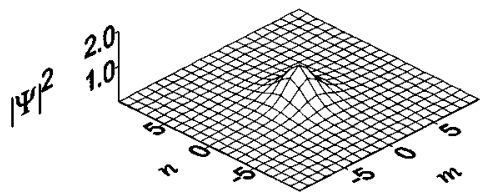

7.0

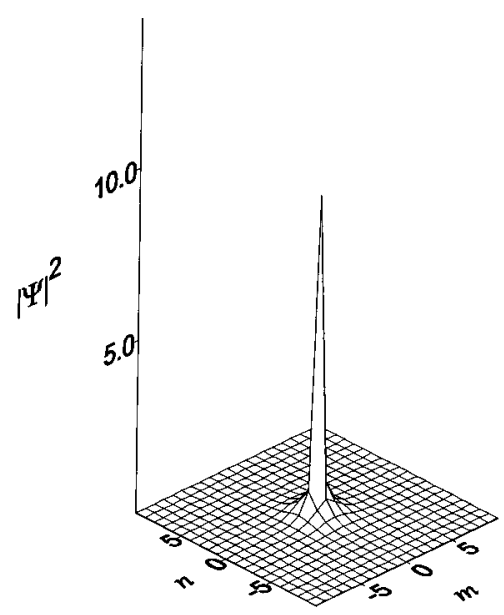

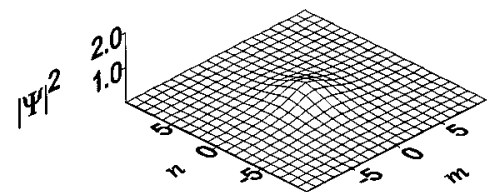

5.0

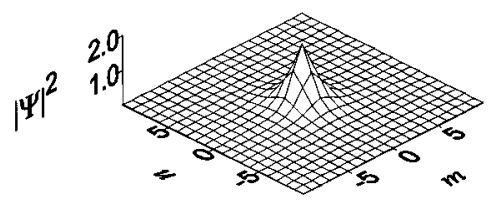

8.0

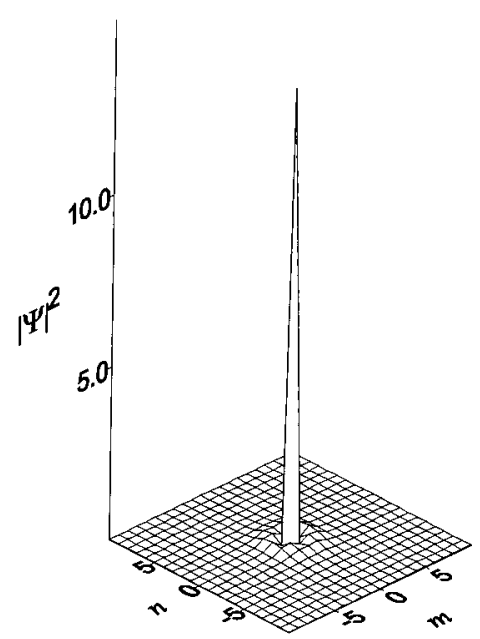

FIG. 13. Same as in Fig. 12, but with a staggered initial Gaussian profile $(K=\pi, Q=0)$.

$$
\left(i \frac{\partial}{\partial t}+\frac{\partial^{2}}{\partial x^{2}}-\frac{\partial^{2}}{\partial y^{2}}\right) \psi+|\psi|^{2} \psi=0
$$

For this case, no localized solution exists, ${ }^{44}$ and an initially localized waveform will never collapse, but elongate in the $y$ direction and narrow in the $x$ direction. ${ }^{45}$ The same behavior can be expected for broad initial conditions in the twodimensional nearest-neighbor discrete NLS equation with different signs in the coupling terms, since it is just a discretization of Eq. (54). In the case considered here, with anisotropic long-range dipole-dipole interaction, we expect that the effective dispersion, as experienced by a broad, unstaggered excitation, can be approximated by the dispersion function $J(k, q)(10)$ around the point $k=q=0$. Since we know from Sec. II that this point is a saddle point, the effective coupling in the $m$ and $n$ directions will have opposite signs, and a similar behavior as for Eq. (54) can be expected.

The dynamical behavior in the isotropic lattice is illustrated in Fig. 14. It appears that the dynamics of the isotropic collapse is characterized by requiring a considerable value of the excitation number for a Gaussian initial condition to ex- hibit quasicollapse. Thus, for the parameter values in Fig. 14 the threshold occurs around $N \simeq 300$. Figure 14(a) shows collapse dynamics $(N \simeq 325)$ leading to considerable and fast localization of the energy in the vicinity of the core, followed by oscillations. Figure 14(b) displays subthreshold dynamics $(N \simeq 250)$ of the initial excitation which spreads out.

The origin of the high threshold value of $N$ in the isotropic case can be understood from the dependence of $N$ on $E$ in the stationary case displayed in Fig. 2(d). Here we note the resemblance to the situation in the three-dimensional cubic NLS equation, which is known to exhibit supercritical collapse behavior. ${ }^{36}$ Using a simple scaling argument it is also evident from the renormalized continuum limit of Eq. (5) in the isotropic case

$$
i \psi_{t}-\sqrt{-\nabla^{2}} \psi+|\psi|^{2} \psi=0
$$

that the collapse dynamics has supercritical characteristics. For the supercritical case the threshold value of $N$ is known to depend on the explicit form of the initial condition ${ }^{46}$ implying that broad initial conditions (as in Fig. 14) require a rather large excitation number for collapse to occur. A de- 


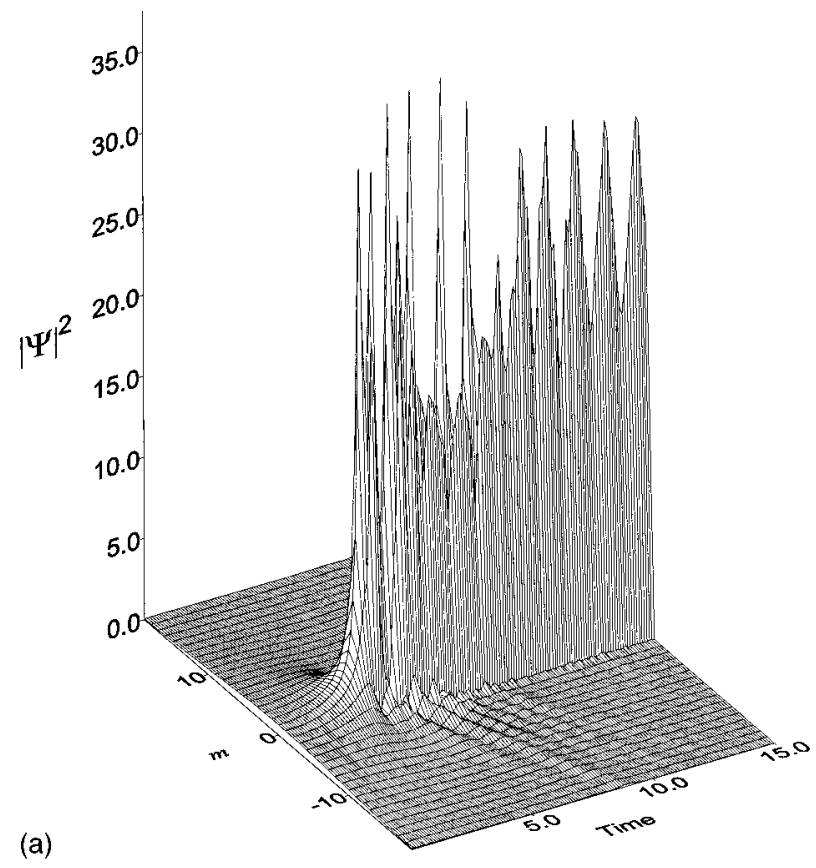

(a)

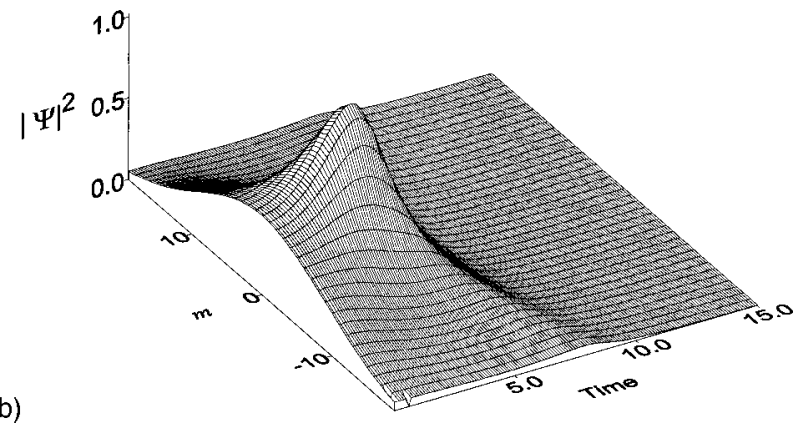

(b)

FIG. 14. Dynamical solution of Eq. (5) $(a=1)$ with isotropic dipole-dipole interaction (4) $[\vec{d}=(0,0,1)]$ and with the Gaussian profile (53) with $K=0, Q=0, m_{0}=n_{0}=18$, and (a) $N=325$ (A $=0.8)$, resp. (b) $N \simeq 250(A \simeq 0.7)$, as initial condition. The figure shows a cut along the axis $n=0$.

tailed investigation of the collapse dynamics in the framework of Eq. (55) will be presented elsewhere.

\section{CONCLUSIONS}

In the present paper we have considered the effects of long-range dispersive coupling of the dipole-dipole type in the two-dimensional discrete nonlinear Schrödinger equation. From a general criterion for modulational instability of plane waves, we have concluded that in the anisotropic case, with in-plane alignment of the dipole moments, modulational instability for long-wavelength perturbations at an extremal point of the dispersion surface always occurs for plane waves that are staggered only in one direction. In the isotropic case, with dipole moments aligned perpendicular to the lattice plane, modulational instability occurs either for waves staggered in both directions (for attractive nonlinearity), or for unstaggered waves (for repulsive nonlinearity). We have found that the broad nonlinear stationary excitations, appearing as a result of the modulational instability, in general are staggered in the same way as the corresponding plane waves. However, in contrast to what is seen in the case of nearestneighbor dispersion, the more narrow stationary states are in general found to be staggered only in a region close to its central site, and asymptotically unstaggered.

Concerning the linear stability of the stationary states, we have found that, similar to the case with nearest-neighbor dispersion, broad excitations in general are unstable, while the narrow, intrinsically localized states are stable. In the case of anisotropic dispersion, we have demonstrated the existence of a threshold for quasicollapse of broad excitations (staggered in one direction) into intrinsically localized modes. On the other hand, broad unstaggered excitations have been found to split and spread. In the isotropic case, quasicollapse is observed, however for much higher excitation numbers.

To make the comparison between the model investigated in this paper and the standard continuum and discrete nearest neighbor NLS model more clear and explicit, we include Table I that summarizes the main differences.

In general we have shown that the existence of staggered localized states is a generic property of nonlinear models with dipole-dipole dispersive interaction. The staggered states have a longer lifetime than the unstaggered states since the process of direct photon emission by the staggered excitation is prohibited due to the momentum conservation law. In this way, in molecular systems with dipole-dipole dispersive interaction the energy may be stored using staggered localized excitations. Our results on the effects of long-range dipole-dipole dispersion in the repulsive NLS equation may be used in describing the dynamics of easy-plane ferromagnetic materials where the vortex dynamics in the case of an external magnetic field perpendicular to the easy-plane is described by the repulsive NLS equation (see, e.g., Ref. 51). Magnetic dipolar forces changing fundamentally the ground state of the two-dimensional Heisenberg ferromagnets (see, e.g., Ref. 52) will also influence the dynamics of these materials in accordance with the results of our investigation presented in this paper.

\section{ACKNOWLEDGMENTS}

Yu.B.G. would like to express his thanks for the hospitality of the Technical University of Denmark, where part of

TABLE I. Comparison of the properties of the dipole-dipole interaction model to the properties of the standard continuum and discrete nearest-neighbor NLS models.

\begin{tabular}{|c|c|c|c|}
\hline Phenomenon & $\begin{array}{c}\text { Discrete } \\
\text { and nonlocal }\end{array}$ & $\begin{array}{l}\text { Standard 2D continuum } \\
\text { continuum }\end{array}$ & $\begin{array}{c}\text { Discrete } \\
\text { nearest neighbor }\end{array}$ \\
\hline Bistability & Present & Not present & Present \\
\hline Staggered states & Stagger in core only & Not present & Stagger globally \\
\hline (Quasi)collapse & Supercritical & Critical & Critical \\
\hline
\end{tabular}


this work was done, and also acknowledges the Ukrainian Fundamental Research Fund under Grant 2.4/355. V.K.M. wishes to thank the Ris $\varnothing$ National Laboratory for hospitality. M.J. acknowledges support from the Swedish Foundation for International Cooperation in Research and Higher Education. This work was supported by the INTAS under Grant Nos. 93-139 and 96-0413 and by the Danish Natural Science Research Council SNF under Grant No. 9600852.

\section{APPENDIX}

In this appendix we derive an approximate formula for the dispersion function

$$
J(\vec{k}) \equiv J(k, q)=\sum_{\vec{m}, \vec{m} \neq 0} \frac{e^{i \vec{k} \cdot \vec{m}}}{|\vec{m}|^{3}}\left[1-3\left(\vec{d} \cdot \frac{\vec{m}}{|\vec{m}|}\right)^{2}\right]
$$

where the summation extends over all integral components of the vector $\vec{m}=(m, n, 0)$ excluding the term with $\vec{m}=\overrightarrow{0}$. First we consider the case when the dipole moments are aligned along the $x$ axis: $\vec{d}=(1,0,0)$. We will consider the behavior of the dispersion function $J(k, q)$ in the vicinity of its minimum $(k=0, q=\pi)$ and its maximum $(k=\pi, q=0)$ separately.

In the vicinity of the minimum $(k=0, q=\pi)$ it is useful to present the dispersion function as follows:

$$
\begin{aligned}
J(k, q)= & 2 \sum_{n=1}^{\infty} \sum_{m=-\infty}^{\infty} \frac{\cos (k m) \cos (q n)}{\left(m^{2}+n^{2}\right)^{3 / 2}}\left(1-3 \frac{m^{2}}{m^{2}+n^{2}}\right) \\
& -4 \operatorname{Re} F\left(e^{i k}, 3\right),
\end{aligned}
$$

where

$$
F(z, s)=\sum_{n=1}^{\infty} \frac{z^{n}}{n^{s}}
$$

is the Jonqière's function. Its properties are described in Ref. 47. Using the Poisson's summation formula

$$
\sum_{m=-\infty}^{\infty} f(2 \pi m)=\sum_{m=-\infty}^{\infty} \frac{1}{2 \pi} \int_{-\infty}^{\infty} d \tau f(\tau) e^{i m \tau},
$$

we obtain

$$
\begin{gathered}
\sum_{m=-\infty}^{\infty} \frac{\cos (k m)}{\left(m^{2}+n^{2}\right)^{3 / 2}}\left(1-3 \frac{m^{2}}{m^{2}+n^{2}}\right) \\
=\sum_{m=-\infty}^{\infty}\left[(2 \pi m-k)^{2} K_{0}(|2 \pi m-k| n)\right. \\
\left.\quad+(2 \pi m+k)^{2} K_{0}(|2 \pi m+k| n)\right],
\end{gathered}
$$

where $K_{\nu}(z)$ is the modified Bessel function. ${ }^{47}$ Then Eq. (A2) can be written as

$$
\begin{aligned}
J(k, q)= & 4 k^{2} \sum_{n=1}^{\infty} \cos (q n) K_{0}(|k| n)+4 \sum_{n=1}^{\infty} \sum_{m=1}^{\infty} \cos (q n) \\
& \times\left[(2 \pi m-k)^{2} K_{0}(|2 \pi m-k| n)\right. \\
& \left.+(2 \pi m+k)^{2} K_{0}(|2 \pi m+k| n)\right]-4 \operatorname{Re} F\left(e^{i k}, 3\right) .
\end{aligned}
$$

For $|k|<1$ the second term in Eq. (A6) is a rapidly converging series $\left[K_{0}(z) \simeq \sqrt{\pi / 2 z} e^{-z}\right.$ when $\left.z \gg 1\right]$. Therefore one can write with a good accuracy

$$
\begin{aligned}
& \sum_{n=1}^{\infty} \sum_{m=1}^{\infty} \cos (q n)\left[(2 \pi m-k)^{2} K_{0}(|2 \pi m-k| n)\right. \\
& \left.\quad+(2 \pi m+k)^{2} K_{0}(|2 \pi m+k| n)\right] \\
& \simeq\left[(2 \pi-k)^{2} K_{0}(2 \pi-k)\right. \\
& \left.\quad+(2 \pi+k)^{2} K_{0}(2 \pi+k)\right] \cos (q) .
\end{aligned}
$$

Taking into account Eq. (A7) and the relations ${ }^{47,48}$

$\sum_{n=1}^{\infty} \cos (q n) K_{0}(|k| n)$

$$
\begin{aligned}
= & \frac{1}{2}\left[\gamma+\ln \left(\frac{|k|}{4 \pi}\right)\right]+\frac{\pi}{2 \sqrt{k^{2}+q^{2}}} \\
& +\frac{\pi}{2} \sum_{m=1}^{\infty}\left(\frac{1}{\sqrt{k^{2}+(2 m \pi-q)^{2}}}-\frac{1}{2 m \pi}\right) \\
& +\frac{\pi}{2} \sum_{m=1}^{\infty}\left(\frac{1}{\sqrt{k^{2}+(2 m \pi+q)^{2}}}-\frac{1}{2 m \pi}\right)
\end{aligned}
$$

$\operatorname{Re} F\left(e^{i k}, 3\right)=\zeta(3)-\frac{k^{2}}{4}\left[3-\ln \left(k^{2}\right)\right]$

$$
+\sum_{m=2}^{\infty} \zeta(3-2 m)(-1)^{m} \frac{k^{2 m}}{(2 m) !}
$$

where $\gamma \simeq 0.577$ is the Euler's constant and $\zeta(x)$ is the Riemann $\zeta$ function, we obtain that for $|k| \ll 1$ and $|q-\pi| \ll 1$

$$
\begin{aligned}
J(k, q) \simeq & -4 \zeta(3)-32 \pi^{2} K_{0}(2 \pi)+[3+2 \gamma-2 \ln (\pi) \\
& -8\left(\pi^{2}+1\right) K_{0}(2 \pi)+32 \pi K_{1}(2 \pi) \\
& \left.-8 \pi^{2} K_{2}(2 \pi)\right] k^{2}+32 \pi^{2} K_{0}(2 \pi)(1+\cos q) \\
\simeq & -5.1+1.79 k^{2}+0.29(1+\cos q)
\end{aligned}
$$

By means of the Poisson's summation formula (A4), one can obtain that in the vicinity of the maximum $(k=\pi, q$ $=0$ ) the dispersion function can be written as 


$$
\begin{aligned}
J(k, q)= & 2 \sum_{m=1}^{\infty} \sum_{n=-\infty}^{\infty} \frac{\cos (k m) \cos (q n)}{\left(m^{2}+n^{2}\right)^{3 / 2}}\left(1-3 \frac{m^{2}}{m^{2}+n^{2}}\right) \\
& +2 \operatorname{Re} F\left(e^{i q}, 3\right) \\
= & -4 q^{2} \sum_{m=1}^{\infty}\left(K_{0}(|q| m)+\frac{K_{1}(|q| m)}{|q| m}\right) \cos (k m) \\
& -4 \sum_{m, n=1}^{\infty}\left[(2 \pi n-q)^{2}\right. \\
& \times\left(K_{0}[(2 \pi n-q) m]+\frac{K_{1}[(2 \pi n-q) m]}{(2 \pi n-q) m}\right) \\
& +i \operatorname{dem}(q \rightarrow-q)] \cos (k m)+2 \operatorname{Re} F\left(e^{i q}, 3\right) .
\end{aligned}
$$

(A11)

Using the formula ${ }^{49}$

$$
\begin{aligned}
\sum_{m=1}^{\infty} \cos (k m) \frac{q}{m} K_{1}(q m) \\
=-\frac{\pi^{2}}{12}+\frac{1}{4}(\pi-k)^{2}+\frac{q^{2}}{8}\left[1+\psi\left(\frac{k}{2 \pi}\right)+\psi\left(1-\frac{k}{2 \pi}\right)\right. \\
\left.+\ln \left(\frac{16 \pi^{2}}{q^{2}}\right)\right]-\frac{\pi}{2} \sum_{m=-\infty}^{\infty}\left(\sqrt{(2 \pi m+k)^{2}+q^{2}}\right. \\
\left.-|2 \pi m+k|-\frac{q^{2}}{2|2 \pi m+k|}\right)
\end{aligned}
$$

and Eqs. (A8) and (A9), we get for $|q| \ll 1$ and $|k-\pi| \ll 1$

$$
\begin{aligned}
J(k, q) \simeq & 2 \zeta(3)+\frac{\pi^{2}}{3}-\left[2+\gamma-\ln \pi-4\left(1+4 \pi^{2}\right) K_{0}(2 \pi)\right. \\
& \left.+8 K_{1}(2 \pi)\right] q^{2}-32 \pi^{2}\left(K_{0}(2 \pi)+\frac{K_{1}(2 \pi)}{2 \pi}\right) \cos k \\
\simeq & 6.03-1.29 q^{2}-(\pi-k)^{2}-0.34(1+\cos k) . \quad(\text { A13 })
\end{aligned}
$$

Let us now consider the isotropic case when the dipole moments are perpendicular to the $(x, y)$ plane $[\vec{d}=(0,0,1)]$, and the dispersion function has the form

$$
J(k, q)=4 \sum_{m, n=1}^{\infty} \frac{\cos (k m) \cos (q n)}{\left(m^{2}+n^{2}\right)^{3 / 2}}
$$

$$
+2 \sum_{m=1}^{\infty} \frac{\cos (k m)+\cos (q m)}{m^{3}} .
$$

The function (A14) has a minimum at $k=q=\pi$ and a maximum at $k=q=0$. It is known ${ }^{50}$ that for $\operatorname{Re} s>0$,

$$
\sum_{m, n=1}^{\infty} \frac{(-1)^{n+m}}{\left(m^{2}+n^{2}\right)^{s}}=\left(1-2^{1-2 s}\right) \zeta(2 s)-\left(1-2^{1-s}\right) \zeta(s) \beta(s),
$$

where $\beta(s)$ is the analytical continuation of the Dirichlet series

$$
\beta(s)=\sum_{m=0}^{\infty} \frac{(-1)^{m}}{(2 m+1)^{s}} .
$$

The properties of $\beta(s)$ were considered in Ref. 50. Thus, in the vicinity of the minimum $k=q=\pi$, the dispersion function can be represented in the form

$$
\begin{aligned}
J(k, q) \simeq & -4\left(1-2^{-1 / 2}\right) \zeta\left(\frac{3}{2}\right) \beta\left(\frac{3}{2}\right)+\left(1-2^{1 / 2}\right) \zeta\left(\frac{1}{2}\right) \beta\left(\frac{1}{2}\right) \\
& \times\left[(\pi-k)^{2}+(\pi-q)^{2}\right] \\
= & -2.646+0.404\left[(\pi-k)^{2}+(\pi-q)^{2}\right] .
\end{aligned}
$$

In the vicinity of the point $k=q=0$, the asymptotic expression for the dispersion function can be obtained directly from Eq. (A1) by replacing the summation over $\vec{m}$ by an integration over the two-dimensional $\vec{m}$ space

$$
J(\vec{k}) \simeq J(\overrightarrow{0})+\int d \vec{m} \frac{\left(e^{i \vec{k} \cdot \vec{m}}-1\right)}{|\vec{m}|^{3}}\left[1-3\left(\vec{d} \cdot \frac{\vec{m}}{|\vec{m}|}\right)^{2}\right] .
$$

As a result we get

$$
\begin{aligned}
J(\vec{k}) \simeq & J(\overrightarrow{0})+2 \pi\left(\frac{(k \cos \phi+q \sin \phi)^{2} \sin ^{2} \theta}{\sqrt{k^{2}+q^{2}}}\right. \\
& \left.-\sqrt{k^{2}+q^{2}} \cos ^{2} \theta\right)
\end{aligned}
$$

where

$$
J(\overrightarrow{0})=4\left(1-\frac{3 \sin ^{2} \theta}{2}\right) \zeta(3 / 2) \beta(3 / 2) \simeq 9.03\left(1-\frac{3 \sin ^{2} \theta}{2}\right) .
$$

*Permanent address: Bogolyubov Institute for Theoretical Physics, $252143 \mathrm{Kiev}$, Ukraine.

†Permanent address: Institute for Automation and Electrometry, 630090, Novosibirsk, Russia.

${ }^{1}$ D. N. Christodoulides and R. I. Joseph, Opt. Lett. 13, 794 (1988).

${ }^{2}$ A. B. Aceves, C. De Angelis, A. M. Rubenchik, and S. K. Turitsyn, Opt. Lett. 19, 329 (1994).
${ }^{3}$ E. W. Laedke, K. H. Spatschek, S. K. Turitsyn, and V. K. Mezentsev, Phys. Rev. E 52, 5549 (1995).

${ }^{4}$ L. M. Floría, J. L. Marín, P. J. Martínez, F. Falo, and S. Aubry, Europhys. Lett. 36, 539 (1996).

${ }^{5}$ A. C. Scott, Phys. Rep. 217, 1 (1992).

${ }^{6}$ S. A. Kiselev, S. R. Bickham, and A. J. Sievers, Comments Condens. Matter Phys. 17, 135 (1995).

${ }^{7}$ M. J. Ablowitz and J. F. Ladik, J. Math. Phys. 17, 1011 
(1976)

${ }^{8}$ A. C. Scott and L. MacNeil, Phys. Lett. 98A, 87 (1983).

${ }^{9}$ A. J. Sievers and S. Takeno, Phys. Rev. Lett. 61, 970 (1988).

${ }^{10}$ V. M. Burlakov, S. A. Kiselev, and V. N. Pyrkov, Phys. Rev. B 42, 4921 (1990).

${ }^{11}$ R. Bourbonnais and R. Maynard, Phys. Rev. Lett. 64, 1397 (1990).

${ }^{12}$ S. R. Bickham and A. J. Sievers, Phys. Rev. B 43, 2339 (1991)

${ }^{13}$ J. C. Eilbeck and R. Flesh, Phys. Lett. A 149, 200 (1990).

${ }^{14}$ T. Dauxois, M. Peyrard, and A. R. Bishop, Phys. Rev. E 47, 684 (1993).

${ }^{15}$ R. S. MacKay and S. Aubry, Nonlinearity 7, 1623 (1994).

${ }^{16}$ S. Flach, K. Kladko, and R. S. MacKay, Phys. Rev. Lett. 78, 1207 (1997)

${ }^{17}$ S. Takeno, J. Phys. Soc. Jpn. 58, 759 (1989).

${ }^{18}$ M. Salerno, Phys. Rev. A 46, 6856 (1992).

${ }^{19}$ D. Cai, A. R. Bishop, and N. Gronbech-Jensen, Phys. Rev. Lett. 72, 591 (1994).

${ }^{20}$ P. L. Christiansen, Yu. B. Gaididei, K. Ф. Rasmussen, V. K. Mezentsev, and J. Juul Rasmussen, Phys. Rev. B 54, 900 (1996).

${ }^{21}$ P. Woafo, J. R. Kenne, and T. C. Kofane, J. Phys.: Condens. Matter 5, L123 (1993).

${ }^{22}$ G. A. Baker, Jr., Phys. Rev. 122, 1477 (1961).

${ }^{23}$ A. M. Kac and B. C. Helfand, J. Math. Phys. 4, 1078 (1972).

${ }^{24}$ L. Vazquez, W. A. B. Evans, and G. Rickayzen, Phys. Lett. A 189, 454 (1994).

${ }^{25}$ Yu. Gaididei, N. Flytzanis, A. Neuper, and F. G. Mertens, Phys. Rev. Lett. 75, 2240 (1995).

${ }^{26}$ O. M. Braun, Yu. S. Kivshar, and I. I. Zelenskaya, Phys. Rev. B 41, 7118 (1990).

${ }^{27}$ A. Dikande and T. C. Kofane, Physica D 83, 450 (1995).

${ }^{28}$ G. L. Alfimov, V. M. Eleonskii, N. E. Kulagin, and N. V. Mitskevich, Chaos 3, 405 (1993).

${ }^{29}$ Yu. B. Gaididei, S. F. Mingaleev, P. L. Christiansen, and K.. . Rasmussen, Phys. Lett. A 222, 152 (1996).

${ }^{30}$ Yu. B. Gaididei, S. F. Mingaleev, P. L. Christiansen, and K.. Rasmussen, Phys. Rev. E 55, 6141 (1997).

${ }^{31}$ A. S. Davydov, Theory of Molecular Excitons (Plenum, New York, 1971).

${ }^{32}$ D. Möbius, Adv. Mater. 7, 437 (1995).
${ }^{33}$ B. A. Ivanov and E. V. Tartakovskaya, Phys. Rev. Lett. 77, 386 (1996).

${ }^{34}$ Yu. S. Kivshar and M. Peyrard, Phys. Rev. A 46, 3198 (1992).

${ }^{35}$ V. I. Petviashvili and O. A. Pokhotelov, Solitary Waves in Plasma and in the Atmosphere (Energoatomizdat, Moscow, 1992); L. A. Abramyan, Yu. A. Stepanyants, and V. L. Shrira, Dokl. Akad. Nauk USSR 327, 460 (1992) [Sov. Phys. Dokl. 37, 575 (1992)].

${ }^{36}$ J. Juul Rasmussen and K. Rypdal, Phys. Scr. 33, 481 (1986).

${ }^{37}$ V. I. Shrira, Dokl. Akad. Nauk SSSR 308, 732 (1989) [in Russian].

${ }^{38}$ A. I. D'yachenko and E. A. Kuznetsov, Physica D 87, 301 (1995).

${ }^{39}$ E. W. Laedke, K. H. Spatschek, V. K. Mezentsev, S. L. Musher, I. V. Ryzhenlova, and S. K. Turitsyn, Pis'ma Zh. Eksp. Teor. Fiz. 62, 652 (1995) [JETP Lett. 62, 677 (1995)].

${ }^{40}$ E. W. Laedke, K. H. Spatschek, and S. K. Turitsyn, Phys. Rev. Lett. 73, 1055 (1994).

${ }^{41}$ V. K. Mezentsev, S. L. Musher, I. V. Ryzhenkova, and S. K. Turitsyn, Pis'ma Zh. Eksp. Teor. Fiz. 60, 815 (1994) [JETP Lett. 60, 829 (1994)].

${ }^{42}$ T. R. Taha and M. J. Ablowitz, J. Comput. Phys. 55, 203 (1984).

${ }^{43}$ P. L. Christiansen, Yu. B. Gaididei, V. K. Mezentsev, S. L. Musher, K. Ф. Rasmussen, J. Juul Rasmussen, I. V. Ryzhenkova, and S. K. Turitsyn, Phys. Scr. T67, 160 (1996).

${ }^{44}$ L. Bergé, E. A. Kuznetsov, and J. Juul Rasmussen, Phys. Rev. E 53, R1340 (1996).

${ }^{45}$ L. Bergé, Phys. Lett. A 189, 290 (1994).

${ }^{46}$ E. A. Kuznetsov, J. Juul Rasmussen, K. Rypdal, and S. K. Turitsyn, Physica D 87, 273 (1995).

${ }^{47}$ W. Magnus, F. Oberhettinger, and R. P. Soni, Formulas and Theorems for the Special Functions of Mathematical Physics (Springer-Verlag, Berlin, 1966).

${ }^{48}$ I. S. Gradshteyn and I. M. Ryzhik, Table of Integrals, Series and Products (Academic, New York, 1965).

${ }^{49}$ S. Allen and R. K. Pathria, J. Math. Phys. 34, 1497 (1992).

${ }^{50}$ M. L. Glasser, J. Math. Phys. 14, 409 (1972).

${ }^{51}$ A. M. Kosevich, B. A. Ivanov, and A. S. Kovalev, Phys. Rep. 194, 117 (1990).

${ }^{52}$ Magnetic Properties of Layered Transition Metal Compounds, edited by L. J. Jongh (Kluwer, Boston, 1990). 